\title{
ON THE HOLONOMY OF CONNECTIONS WITH SKEW-SYMMETRIC TORSION
}

\author{
ILKA AGRICOLA AND THOMAS FRIEDRICH
}

\begin{abstract}
We investigate the holonomy group of a linear metric connection with skew-symmetric torsion. In case of the euclidian space and a constant torsion form this group is always semisimple. It does not preserve any non-degenerated 2 -form or any spinor. Suitable integral formulas allow us to prove similar properties in case of a compact Riemannian manifold equipped with a metric connection of skew-symmetric torsion. On the Aloff-Wallach space $N(1,1)$ we construct families of connections admitting parallel spinors. Furthermore, we investigate the geometry of these connections as well as the geometry of the underlying Riemannian metric. Finally, we prove that any 7-dimensional 3-Sasakian manifold admits $\mathbb{P}^{2}$-parameter families of linear metric connections and spinorial connections defined by 4 -forms with parallel spinors.
\end{abstract}

\section{Contents}

1. Introduction 1

2. The eight classes of linear connections with torsion 3

3. The holonomv of spinor connections with constant torsion in $\mathbb{R}^{n} \quad 5$

4. Constant 3-forms in $\mathbb{R}^{n}$ and their holonomv algebra 8

5. $\quad \nabla$-parallel 2-forms on manifolds 12

6. Schrödinger-Lichnerowicz tvpe formulas for Dirac operators 13

7. 1-parameter families of connections with parallel spinors 15

8. Torsion forms with parallel spinors on Aloff-Wallach spaces 19

9. Torsion forms with parallel spinors on 3-Sasakian manifolds 26

Appendix A. The Lie algebra $\mathfrak{s p i n}(9)$ inside $\mathfrak{s o}(16) \quad 28$

$\begin{array}{ll}\text { References } & 29\end{array}$

\section{Introduction}

This paper treats the geometry of metric invariant connections with skew-symmetric torsion, as they became recently of interest in string theory and special geometries. The notion of torsion of a connection was invented by Elie Cartan, and appeared for the first time in a short note at the Académie des Sciences de Paris in 1922 (see [6] $)^{1}$. Although it contains no formulas, Cartan observes that such a connection may or may not preserve geodesics, and turns his attention first to those who actually do so. In this sense, E. Cartan was the first to investigate this class of connections. At that time,

Received by the editors 22nd October 2018.

2000 Mathematics Subject Classification. Primary 53 C 25; Secondary 81 T 30.

Key words and phrases. Parallel spinors, connections with skew-symmetric torsion, string theory.

Supported by the SFB 288 "Differential geometry and quantum physics" of the DFG.

${ }^{1}$ We thank Andrzej Trautman for drawing our attention to these papers by Cartan - see [23]. 
it was not yet customary - as it became later in the second half of the 20th century - to assign to a Riemannian manifold only its Levi-Civita connection. Rather, Cartan demands (see [9]):

Étant donné une variété plongée dans l'espace affine (ou projectif, ou conforme etc.), attribuer à cette variété la connexion affine (ou projective, ou conforme etc.) qui rende le plus simplement compte des relations de cette variété avec l'espace ambiant.

He then goes on to explain in very general terms how the connection should be adapted to the geometry under consideration. This point of view should be taken into account in Riemannian geometry, too. The canonical connection of a naturally reductive Riemannian space is a first example (see [1]). Moreover, we know many non integrable geometric structures on Riemannian manifolds admitting a unique metric connection preserving the structure and with non vanishing skew-symmetric torsion (see [15, 14]). Following Cartan as well as the idea that torsion forms are candidates for the so called $B$-field in string theory, the geometry of these connections deserves systematic investigation. Basically, there are no general results concerning the holonomy group of connections with torsion. The question whether or not a connection of that type admits parallel tensor fields differs radically from the corresponding problem for the Levi-Civita connection. In particular, one is interested in the existence of parallel spinor fields, since they are interpreted in string theory as supersymmetries of the model.

The paper is organized as follows. In Section 2, we discuss once again some basic results motivating the role of metric connections with skew-symmetric torsion. In Sections 3 and 4, we study the linear case, i.e., euclidian space equipped with a constant torsion form $\mathrm{T}$. The holonomy algebra $\mathfrak{g}_{\mathrm{T}}^{*}$ of the corresponding linear connection has some remarkable properties. For any 3 -form, $\mathfrak{g}_{\mathrm{T}}^{*}$ is a semisimple Lie algebra. Moreover, it cannot preserve a non degenerate 2 -form or a spinor. On the other side, many representations of a compact, semisimple Lie algebra occur as the holonomy algebra of some 3-form, for example the adjoint representation can be realized in this way. We introduce an obstruction for a Lie algebra representation to be the holonomy algebra of some 3-form and show on an example how it may be used to rule out some representations. In particular, the unique, irreducible 16-dimensional representation of the algebra $\mathfrak{s p i n}(9)$ cannot be the holonomy algebra of some 3 -form. Forms of higher degree than three do not occur for linear connections, but they define spinorial connections. In the euclidian case we introduce their spinorial holonomy algebra as a Lie subalgebra of the Clifford algebra. In all examples discussed, this algebra turns out to be perfect.

In Section 5 and 6, we generalize the algebraic results to the case of a Riemannian manifold $\left(M^{n}, g, \mathrm{~T}\right)$ with a metric connection $\nabla$. In particular, we are interested in the question whether or not the $\nabla$-holonomy group preserves a spinor field. In the compact case, we prove that if the scalar curvature $\mathrm{Scal}^{g} \leq 0$ is non positive and if the torsion form is closed, $d \mathrm{~T}=0$, any $\nabla$-parallel spinor is Riemannian parallel and $\mathrm{T}=0$ vanishes. Here we use an integral formula for the square of the Dirac operator depending on the connection. The main point is that the formula becomes simple if one compares the Dirac operator corresponding the connection with torsion form $\mathrm{T}$ with the spinorial Laplace operator corresponding to the connection with torsion form $3 \cdot \mathrm{T}$. This effect has been observed in the literature at several places, in particular by Bismut (see [3]) and, in the homogeneous case, by Agricola (see [1]). We explore the corresponding integral formula and study the space of parallel spinors. 
In Section 7 we discuss, for a given triple $\left(M^{n}, g, \mathrm{~T}\right)$, the whole family $\nabla^{s}$ of metric connections with torsion form $s \cdot \mathrm{T}$. In the generic case, the existence of a $\nabla^{s}$-parallel spinor restricts the possible parameter $s$ via a polynomial equation. Consequently, in the generic case, at most a finite number of connections in the family admits parallel spinors. Some simple examples show that sometimes two connections really admit parallel spinors. Moreover, our integral formulas prove that, on a compact manifold, basically only three parameters are possible. In case that the torsion form is associated with a special non integrable geometry, the connection $\nabla^{s}$ with a parallel spinor is sometimes unique. A result of that type requires additional informations concerning the underlying geometry. We prove it for 5-dimensional Sasakian manifolds equipped with their canonical connection.

In Section 8 we construct, on the Aloff-Wallach manifold $N(1,1)=\mathrm{SU}(3) / S^{1}$, a twoparameter family of metrics that admits two inequivalent cocalibrated $G_{2}$-structures. Moreover, we investigate the torsion forms of their unique connections as well as other geometric data of these connections. Our approach is different from the usual one (see [5]). First we construct 3 -forms with parallel spinors on $N(1,1)$. The underlying $\mathrm{G}_{2^{-}}$ structure is cocalibrated and many of the geometric data are encoded into the torsion 3 -form we started with. Moreover, we are interested not only in the type of the $\mathrm{G}_{2^{-}}$ structure, but mainly in the geometry of the unique connection preserving this structure. The same method is then applied in order to construct spinorial connections defined by 4form and admitting parallel spinor fields. Some of these connections are closely related to the 3 -Sasakian structure of $N(1,1)$. In section 9 we generalize these examples. Indeed, we are able to construct, for any 7-dimensional 3-Sasakian manifold, a canonical $\mathbb{P}^{2}$ parameter family of 3 - and 4-forms such that the underlying linear or spinorial connection admits parallel spinors.

\section{The eight classes of linear connections with torsion}

We begin by an elementary, yet enlightening investigation of geometric torsion tensors. Consider a Riemannian manifold $\left(M^{n}, g\right)$. In a point, the difference between its LeviCivita connection $\nabla^{g}$ and any linear connection $\nabla$ is a $(2,1)$-tensor $A$,

$$
\nabla_{X} Y=\nabla_{X}^{g} Y+A(X, Y), \quad X, Y \in T M
$$

The vanishing of the symmetric or the antisymmetric part of $A$ has an immediate geometric interpretation:

Lemma 2.1. The connection $\nabla$ is torsion-free if and only if $A$ is symmetric. The connection $\nabla$ has the same geodesics as the Levi-Civita connection $\nabla^{g}$ if and only if $A$ is antisymmetric.

Proof. The torsion $\mathrm{T}$ of $\nabla$ is

$$
\mathrm{T}(X, Y):=\nabla_{X} Y-\nabla_{Y} X-[X, Y]=A(X, Y)-A(Y, X),
$$

since $\nabla^{g}$ is torsion-free. Hence the first claim follows. For the second, consider a curve $\gamma$ through a point $p$, and set $X:=\dot{\gamma}(p)$. Then

$$
\nabla_{X} X=\nabla_{X}^{g} X+A(X, X),
$$

and hence $\nabla_{X} X$ coincides with $\nabla_{X}^{g} X$ if and only if $A$ is skew-symmetric. 
Following Cartan (see [8, p.51]), we study the algebraic types of the torsion tensor for a metric connection. Denote by the same symbol the $(3,0)$-tensors derived from $A, \mathrm{~T}$ by contraction with the metric,

$$
A(X, Y, Z):=g(A(X, Y), Z), \quad \mathrm{T}(X, Y, Z):=g(\mathrm{~T}(X, Y), Z) .
$$

We identify $T M$ with $T M^{*}$ via the metric from now on. Let $\mathcal{T}$ be the $n^{2}(n-1) / 2$ dimensional space of all possible torsion tensors,

$$
\mathcal{T}=\left\{\mathrm{T} \in \otimes^{3} T M \mid \mathrm{T}(X, Y, Z)=-\mathrm{T}(Y, X, Z)\right\} \cong \wedge^{2} T M \otimes T M .
$$

On the other side, a connection $\nabla$ is metric if and only if and only if $A$ belongs to the space

$$
\mathcal{A}^{g}:=T M \otimes \wedge^{2} T M=\left\{A \in \otimes^{3} T M \mid A(X, V, W)+A(X, W, V)=0\right\} .
$$

The real orthogonal group $\mathrm{O}(n, \mathbb{R})$ acts on both tensor representations $\mathcal{T}$ and $\mathcal{A}^{g}$ in a natural way by $g \cdot \mathrm{T}(X, Y, Z):=\mathrm{T}\left(g^{-1} X, g^{-1} Y, g^{-1} Z\right)$ for $g \in \mathrm{O}(n, \mathbb{R})$.

Proposition 2.1. For $n \geq 3$, the space $\mathcal{T}$ of possible torsion tensors splits under $\mathrm{O}(n, \mathbb{R})$ into the sum of three irreducible representations, $\mathcal{T} \cong T M \oplus \wedge^{3} T M \oplus \mathcal{T}^{\prime}$, as does $\mathcal{A}^{g}$. Furthermore, an equivariant bijection $\Phi: \mathcal{A}^{g} \rightarrow \mathcal{T}$ is given by $\left(A \in \mathcal{A}^{g}, \mathrm{~T} \in \mathcal{T}\right)$

$$
\begin{aligned}
\Phi(A)(X, Y, Z) & =A(X, Y, Z)-A(Y, X, Z), \\
2 \Phi^{-1}(\mathrm{~T})(X, Y, Z) & =\mathrm{T}(X, Y, Z)-\mathrm{T}(Y, Z, X)+\mathrm{T}(Z, X, Y) .
\end{aligned}
$$

The map $\Phi$ is a multiple of the identity precisely on $\wedge^{3} T M$.

Proof. It is clear that $\mathcal{T}$ and $\mathcal{A}^{g}$ split into the same irreducible summands under $\mathrm{O}(n, \mathbb{R})$. Hence, we concentrate on $\mathcal{T}$. There exist two $\mathrm{O}(n, \mathbb{R})$-equivariant contractions from $\mathcal{T}$ into irreducible $\mathrm{O}(n, \mathbb{R})$-representations,

$$
\Phi_{1}: \mathcal{T} \longrightarrow \wedge^{3} T M, \quad \Phi_{2}: \mathcal{T} \longrightarrow T M,
$$

given by

$$
\Phi_{1}(\mathrm{~T})=\frac{1}{3} \mathfrak{S} \mathrm{T}(X, Y, Z), \quad \Phi_{2}(\mathrm{~T})=\sum_{i=1}^{n} \mathrm{~T}\left(e_{i+1}, e_{i}, e_{i+1}\right) e_{i}
$$

Here, $\mathfrak{S}$ denotes antisymmetrisation with respect to all arguments and $e_{1}, \ldots, e_{n}$ is any orthonormal basis of $T M$. Vice versa, $T M$ can be realized as an irreducible subspace of $\mathcal{T}$ via $\Phi_{2}^{-1}: T M \rightarrow \mathcal{T}$

$$
V \mapsto \mathrm{T}_{V}, \mathrm{~T}_{V}(X, Y, Z):=g(X, Z) g(V, Y)-g(Y, Z) g(V, X)
$$

All in all, we identified two irreducible summands of $\mathcal{T}, \wedge^{3} T M \subset \operatorname{ker} \Phi_{2}$ and $T M \subset$ ker $\Phi_{1}$. A dimensional argument shows that $\mathcal{T}^{\prime}:=\operatorname{ker} \Phi_{1} \cap \operatorname{ker} \Phi_{2}$ is not empty. In fact, one easily checks that it is irreducible under the action of $\mathrm{O}(n, \mathbb{R})$, and a routine calculation proves all claims about the isomorphism $\Phi$.

The eight classes of linear connections are now defined by the possible parts of their torsions $\mathrm{T}$ in these components. If one looks at the class of linear metric connections, then these are also uniquely determined by their torsion, since $\Phi^{-1}$ reconstructs $A$ from T. For general connections, T determines $A$ only up to a contribution from the complement of $\mathcal{A}^{g}$ inside $\otimes^{3} T M$, that is, from $T M \otimes S^{2} T M$. Since this space splits itself into two irreducible subspaces, one might as well speak of a total of 16 classes in the general situation. The nice lecture notes by Tricerri and Vanhecke 24] use a similar approach in order to classify homogeneous spaces by the algebraic properties of the torsion of the 
canonical connection. They construct homogeneous examples of all classes, and study their "richness".

The described decompositions shows that a natural class of non-torsion free metric connections are those with skew-symmetric torsion form. We obtain a geometric characterization of these connections.

Corollary 2.1. A connection $\nabla$ on $M$ is metric and geodesics preserving precisely if its torsion $\mathrm{T}$ lies in $\wedge^{3} T M$. In this case, $2 \cdot A=\mathrm{T}$ holds,

$$
\nabla_{X} Y=\nabla_{X}^{g} Y+\frac{1}{2} \cdot \mathrm{T}(X, Y,-),
$$

and the $\nabla$-Killing vector fields coincide with the Riemannian Killing vector fields.

Proof. If $\nabla$ preserves geodesics, $2 \cdot A=\mathrm{T}$ by Lemma 2.1. If $\nabla$ is also metric, $A$ needs in addition to lie in the component of $\mathcal{A}^{g}$ that yields a torsion proportional to $A$, which is $\wedge^{3} T M$ by Proposition 2.1

\section{The holonomy of spinor connections with constant torsion in $\mathbb{R}^{n}$}

We consider the euclidian vector space $\mathbb{R}^{n}$ equipped with its standard inner product. The exterior algebra $\Lambda^{*}\left(\mathbb{R}^{n}\right)$ and the Clifford algebra $\mathrm{Cl}\left(\mathbb{R}^{n}\right)$ are - treated as vector spaces only - equivalent $\mathrm{SO}(n)$-representations. Denote by $\Delta_{n}$ the complex vector space of all $n$-dimensional spinors. The Clifford algebra - and henceforth the exterior algebra, too - acts on $\Delta_{n}$. We denote by $\mathrm{T} \cdot \psi$ the corresponding action of a $k$-form $\mathrm{T}$ on a spinor $\psi \in \Delta_{n}$. It is $\mathrm{SO}(n)$-equivariant and called the Clifford multiplication of a spinor by a $k$-form. The Clifford algebra is an associative algebra and there is an underlying Lie algebra structure,

$$
[\alpha, \beta]=\alpha \cdot \beta-\beta \cdot \alpha, \quad \alpha, \beta \in \mathrm{Cl}\left(\mathbb{R}^{n}\right) .
$$

We denote the corresponding Lie algebra by $\mathfrak{c l}\left(\mathbb{R}^{n}\right)$. The Lie algebra $\mathfrak{s o}(n)$ of the special orthogonal group is a subalgebra of $\mathfrak{c l}\left(\mathbb{R}^{n}\right)$,

$$
\mathfrak{s o}(n)=\operatorname{Lin}\left\{X \cdot Y: X, Y \in \mathbb{R}^{n} \text { and }\langle X, Y\rangle=0\right\} \subset \mathfrak{c l}\left(\mathbb{R}^{n}\right) .
$$

Consider an algebraic $k$-form $\mathrm{T} \in \Lambda^{k}\left(\mathbb{R}^{n}\right)$ and denote by $\mathrm{G}_{\mathrm{T}}$ the group of all orthogonal transformation of $\mathbb{R}^{n}$ preserving the form $\mathrm{T}$. Let $\mathfrak{g}_{\mathrm{T}}$ be its Lie algebra. We associate with any exterior form its covariant derivative $\nabla^{\mathrm{T}}$ acting on spinor fields $\psi: \mathbb{R}^{n} \rightarrow \Delta_{n}$ by the formula

$$
\left.\nabla_{X}^{\mathrm{T}} \psi:=\nabla_{X}^{g} \psi+(X\lrcorner \mathrm{T}\right) \cdot \psi \cdot
$$

Here, $\nabla^{g}$ denotes the Levi-Civita connection. For a 3 -form $\mathrm{T} \in \Lambda^{3}\left(\mathbb{R}^{n}\right)$, the spinorial covariant derivative $\nabla^{\mathrm{T}}$ is induced by a linear metric connection with torsion tensor $2 \cdot \mathrm{T}$,

$$
\nabla_{X}^{\mathrm{T}} Y:=\nabla_{X}^{g} Y+2 \cdot \mathrm{T}(X, Y,-) .
$$

For a general exterior form $\mathrm{T}$, we introduce a new Lie algebra $\mathfrak{g}_{\mathrm{T}}^{*}$ that is a subalgebra of $\mathfrak{c l}\left(\mathbb{R}^{n}\right)$.

Definition 3.1. Let $T$ be an exterior form on $\mathbb{R}^{n}$. The Lie algebra $\mathfrak{g}_{\mathrm{T}}^{*}$ is the subalgebra of $\mathfrak{c l}\left(\mathbb{R}^{n}\right)$ generated by all elements $\left.X\right\lrcorner \mathrm{T}$, where $X \in \mathbb{R}^{n}$ is a vector.

The Lie algebra $\mathfrak{g}_{\mathrm{T}}^{*}$ is invariant under the action of the isotropy group $\mathrm{G}_{\mathrm{T}}$. The derived algebra $\left[\mathfrak{g}_{\mathrm{T}}^{*}, \mathfrak{g}_{\mathrm{T}}^{*}\right]$ is the Lie algebra generated by all curvature transformations of the spinorial connection $\nabla^{\mathrm{T}}$. It is the Lie algebra of the infinitesimal holonomy group of the spinorial covariant derivative $\nabla^{\mathrm{T}}$ (see [20], Chapter II, Section 10): 
Definition 3.2. Let $\mathrm{T}$ be an exterior form on $\mathbb{R}^{n}$. The Lie algebra

$$
\mathfrak{h}_{\mathrm{T}}^{*}:=\left[\mathfrak{g}_{\mathrm{T}}^{*}, \mathfrak{g}_{\mathrm{T}}^{*}\right] \subset \mathfrak{c l}\left(\mathbb{R}^{n}\right)
$$

is called the infinitesimal holonomy algebra of the exterior form $\mathrm{T}$.

The Lie algebra $\mathfrak{h}_{\mathrm{T}}^{*}$ is invariant under the action of the isotropy group $\mathrm{G}_{\mathrm{T}}$, too. For a 3 -form $\mathrm{T}$, the Lie algebras $\mathfrak{g}_{\mathrm{T}}^{*}, \mathfrak{h}_{\mathrm{T}}^{*} \subset \mathfrak{s o}(n)$ are subalgebras of the Lie algebra of the orthogonal group. This inclusion reflects again the fact that the corresponding spinor derivative $\nabla^{\mathrm{T}}$ is induced by a linear metric connection. The following proposition generalizes this observation.

Proposition 3.1. If $\mathrm{T}$ is a $k$-form with $k+\left(\begin{array}{c}k-1 \\ 2\end{array}\right) \equiv 0 \bmod 2$, then $\mathfrak{g}_{\mathrm{T}}^{*}$ is a compact Lie algebra.

Proof. We consider the complex spin representation of the Clifford algebra. There exists a hermitian product on $\Delta_{n}$ such that

$$
\left(X \cdot \psi, \psi_{1}\right)+\left(\psi, X \cdot \psi_{1}\right)=0
$$

for all vectors $X \in \mathbb{R}^{n}$ and all spinors $\psi, \psi_{1} \in \Delta_{n}$. Then, under the condition for the degree of the form $\mathrm{T}$, all endomorphisms $X\lrcorner \mathrm{T}$ acting on $\Delta_{n}$ are skew-symmetric.

The following proposition is a special case of the general holonomy theory. For completeness, let us sketch its proof.

Proposition 3.2. There exists a non-trivial $\nabla^{\mathrm{T}}$-parallel spinor field $\psi: \mathbb{R}^{n} \rightarrow \Delta_{n}$,

$$
\left.\nabla_{X}^{\mathrm{T}} \psi=X(\psi)+(X\lrcorner \mathrm{T}\right) \cdot \psi=0,
$$

if and only if there exists a constant spinor $\psi_{0} \in \Delta_{n}$ such that $\mathfrak{h}_{\mathrm{T}}^{*} \cdot \psi_{0}=0$.

Proof. If $\psi: \mathbb{R}^{n} \rightarrow \Delta_{n}$ is $\nabla^{\mathrm{T}}$-parallel, we differentiate it twice with respect to arbitrary vectors $X, Y \in \mathbb{R}^{n}$. Then we obtain the condition

$$
[X\lrcorner \mathrm{T}, Y\lrcorner \mathrm{T}] \cdot \psi=0,
$$

i.e., $\mathfrak{h}_{\mathrm{T}}^{*} \cdot \psi=0$. Conversely, if $\psi_{0} \in \Delta_{0}$ is a spinor such that $\mathfrak{h}_{\mathrm{T}}^{*} \cdot \psi_{0}=0$, we define the spinor field $\psi: \mathbb{R}^{n} \rightarrow \Delta_{n}$ by the formula

$$
\psi(m):=\operatorname{Exp}(-m\lrcorner \mathrm{T}) \cdot \psi_{0}, \quad m \in \mathbb{R}^{n} .
$$

An easy computation yields that $X(\psi)(m)+(X\lrcorner \mathrm{T}) \cdot \psi(m)$ is given by the formula

$$
\operatorname{Ad}(\operatorname{Exp}(m\lrcorner \mathrm{T}))\left(\frac{[m\lrcorner \mathrm{T}, X\lrcorner \mathrm{T}]}{2}+\frac{[m\lrcorner \mathrm{T},[m\lrcorner \mathrm{T}, X\lrcorner \mathrm{T}]]}{6}+\cdots\right) \cdot \psi_{0} .
$$

The commutators $[m\lrcorner \mathrm{T}, X\lrcorner \mathrm{T}]$ etc. are in $\mathfrak{h}_{\mathrm{T}}^{*}$ and the adjoint action $\left.\operatorname{Ad}(\operatorname{Exp}(m\lrcorner \mathrm{T})\right)$ preserves the holonomy algebra $\mathfrak{h}_{\mathrm{T}}^{*}$ since $\left.m\right\lrcorner \mathrm{T} \in \mathfrak{g}_{\mathrm{T}}^{*}$.

Corollary 3.1. Let $\mathrm{T}$ be an exterior form such that the Lie algebra $\mathfrak{g}_{\mathrm{T}}^{*}$ is perfect, $\mathfrak{h}_{\mathrm{T}}^{*}=$ $\mathfrak{g}_{\mathrm{T}}^{*}$. Then any $\nabla^{\mathrm{T}}$-parallel spinor field $\psi: \mathbb{R}^{n} \rightarrow \Delta_{n}$,

$$
\left.\nabla_{X}^{\mathrm{T}} \psi=X(\psi)+(X\lrcorner \mathrm{T}\right) \cdot \psi=0,
$$

is constant and $\mathfrak{g}_{\mathrm{T}}^{*} \cdot \psi=0$.

Proof. Any parallel spinor field satisfies the condition $\mathfrak{h}_{\mathrm{T}}^{*} \cdot \psi=0$. By assumption, we obtain $\mathfrak{g}_{\mathrm{T}}^{*} \cdot \psi=0$ and the differential equation yields $X(\psi)=0$, i.e., $\psi$ is constant. 
Example 3.1. If $T \in \Lambda^{1}\left(\mathbb{R}^{n}\right)$ is a 1-form, the Lie algebra $\mathfrak{g}_{\mathrm{T}}^{*}$ is generated by one element $1 \in \mathfrak{c l}\left(\mathbb{R}^{n}\right)$ and $\mathfrak{g}_{\mathrm{T}}^{*}=\mathbb{R}, \mathfrak{h}_{\mathrm{T}}^{*}=0$. The general solution of the equation $\nabla^{\mathrm{T}} \psi=0$ is

$$
\psi(m)=e^{-\langle m, \mathrm{~T}\rangle} \cdot \psi_{0}, \quad m \in \mathbb{R}^{n},
$$

where $\psi_{0}$ is constant.

We denote by $e_{1}, \ldots, e_{n}$ an orthonormal frame on $\mathbb{R}^{n}$, and abbreviate as $e_{i j k \ldots}$ the exterior product $e_{i} \wedge e_{j} \wedge e_{k} \wedge \ldots$ of 1 -forms.

Example 3.2. Any 2 -form $\mathrm{T} \in \Lambda^{2}\left(\mathbb{R}^{n}\right)$ of rank $2 k$ is equivalent to $A_{1} \cdot e_{12}+\cdots+A_{k}$. $e_{2 k-1,2 k}$. The Lie algebra $\mathfrak{g}_{\mathrm{T}}^{*}$ is generated by the elements $e_{1}, e_{2}, \cdots, e_{2 k-1}, e_{2 k}$. It is isomorphic to the Lie algebra $\mathfrak{s p i n}(2 k+1)$. In particular, if $n=8$ then $\Delta_{8}=\mathbb{R}^{16}$ is a real, 16-dimensional and the spinorial holonomy algebra of a generic 2 -form in eight variables is the unique 16-dimensional irreducible representation of $\mathfrak{s p i n}(9)$.

Example 3.3. Consider the 4-form $\mathrm{T}=e_{1234}+e_{3456} \in \Lambda^{4}\left(\mathbb{R}^{6}\right)$. The Clifford algebra $\mathrm{Cl}\left(\mathbb{R}^{6}\right)=\operatorname{End}\left(\mathbb{R}^{8}\right)$ is isomorphic to the algebra of all endomorphisms of an 8-dimensional real vector space and $\mathfrak{g}_{\mathrm{T}}^{*}$ is the Lie algebra generated by the elements

$$
e_{234}, \quad e_{134}, \quad e_{124}+e_{456}, \quad e_{123}+e_{356}, \quad e_{346}, \quad e_{345} .
$$

A computation of the whole Lie algebra yields the result that $\mathfrak{g}_{\mathrm{T}}^{*}$ is isomorphic to the Lie algebra $\mathfrak{e}(6)$ of the euclidian group.

Example 3.4. Consider the volume form $\mathrm{T}=e_{123456}$ in $\mathbb{R}^{6}$. The subalgebra $\mathfrak{g}_{\mathrm{T}}^{*}$ of $\mathrm{Cl}\left(\mathbb{R}^{6}\right)=\operatorname{End}\left(\mathbb{R}^{8}\right)$ is isomorphic to the compact Lie algebra $\mathfrak{s p i n}(7)$. Indeed, it is generated by the Lie algebra $\mathfrak{s p i n}(6)$ and all elements of degree five.

Example 3.5. Let us discuss the holonomy algebra of a more complicated 4 -form in seven variables,

$$
\mathrm{T}=e_{12} \cdot\left(e_{34}-e_{56}\right)-e_{17} \cdot\left(e_{45}-e_{36}\right)-e_{27} \cdot\left(e_{35}+e_{46}\right)-e_{3456} .
$$

The 7-dimensional spin representation is real and we describe the holonomy algebra $\mathfrak{g}_{\mathrm{T}}^{*}$ using the spin representation $\mathfrak{c l}\left(\mathbb{R}^{7}\right) \rightarrow \mathfrak{g l}\left(\Delta_{7}\right)=\mathfrak{g l}\left(\mathbb{R}^{8}\right)$ of the Clifford algebra. For this purpose, we introduce the matrices

$$
A_{1}:=\left[\begin{array}{cccc}
0 & 0 & 0 & 0 \\
0 & 0 & 0 & 0 \\
1 & 0 & 0 & 0 \\
0 & 0 & 0 & 0
\end{array}\right], A_{2}:=\left[\begin{array}{cccc}
0 & 0 & 0 & 0 \\
0 & 0 & 0 & 0 \\
0 & 1 & 0 & 0 \\
0 & 0 & 0 & 0
\end{array}\right], A_{3}:=\left[\begin{array}{llll}
0 & 0 & 0 & 0 \\
0 & 0 & 0 & 0 \\
0 & 0 & 1 & 0 \\
0 & 0 & 0 & 0
\end{array}\right], A_{4}:=\left[\begin{array}{llll}
0 & 0 & 0 & 0 \\
0 & 0 & 0 & 0 \\
0 & 0 & 0 & 1 \\
0 & 0 & 0 & 0
\end{array}\right] .
$$

The holonomy algebra, treated as a subalgebra of $\mathfrak{g l}\left(\mathbb{R}^{8}\right)$, is the Lie algebra generated by the following seven matrices:

$$
\begin{gathered}
B_{1}:=\left[\begin{array}{cc}
0 & A_{1} \\
A_{1}^{t} & 0
\end{array}\right], B_{2}:=\left[\begin{array}{cc}
0 & A_{2} \\
A_{2}^{t} & 0
\end{array}\right], B_{3}:=\left[\begin{array}{cc}
0 & A_{3} \\
A_{3}^{t} & 0
\end{array}\right], B_{4}:=\left[\begin{array}{cc}
0 & A_{4} \\
A_{4}^{t} & 0
\end{array}\right], \\
B_{5}:=\left[\begin{array}{cc}
A_{1}+A_{1}^{t} & 0 \\
0 & 0
\end{array}\right], \quad B_{6}:=\left[\begin{array}{cc}
A_{2}+A_{2}^{t} & 0 \\
0 & 0
\end{array}\right], B_{7}:=\left[\begin{array}{cc}
A_{4}+A_{4}^{t} & 0 \\
0 & 0
\end{array}\right] .
\end{gathered}
$$

An investigation of the commutators of these matrices yields the result that $\mathfrak{g}_{\mathrm{T}}^{*}$ is a 46-dimensional subalgebra of $\mathfrak{g l}\left(\mathbb{R}^{8}\right)$,

$$
\mathfrak{g}_{\mathrm{T}}^{*}=\left\{\left[\begin{array}{cc}
X & A \\
A^{t} & Y
\end{array}\right]: X, Y \in \mathfrak{s l}\left(\mathbb{R}^{4}\right) \text { and } A \in \mathfrak{g l}\left(\mathbb{R}^{4}\right)\right\}
$$

No spinor is fixed by the holonomy group of the connection $\nabla^{\mathrm{T}}$, i.e., in the flat space $\nabla^{\mathrm{T}}$-parallel spinors do not exist. Later we will see that this torsion form occurs in certain 
compact Riemannian manifolds in a natural way. On these non flat spaces there exist $\nabla^{\mathrm{T}}$-parallel spinors, see Theorem 9.2 .

\section{Constant 3-forms in $\mathbb{R}^{n}$ and their holonomy algebra}

We will study 3 -forms $\mathrm{T} \in \Lambda^{3}\left(\mathbb{R}^{n}\right)$ and their Lie algebras $\mathfrak{g}_{\mathrm{T}}^{*}$. To begin with, let us consider some examples.

Example 4.1. This is the place to discuss Cartan's first example of a space with torsion (see [6, p. 595]). Consider $\mathbb{R}^{3}$ with its usual euclidian metric, and the connection

$$
\nabla_{X} Y=\nabla_{X}^{g} Y-X \times Y
$$

corresponding, of course, to the choice $\mathrm{T}=-2 \cdot e_{1} \wedge e_{2} \wedge e_{3}$. Cartan observed correctly that this connection has same geodesics than $\nabla^{g}$, but induces a different parallel transport ${ }^{2}$. Indeed, consider the $z$-axis $\gamma(t)=(0,0, t)$, a geodesic, and the vector field $V$ which, in every point $\gamma(t)$, consists of the vector $(\cos t, \sin t, 0)$. Then one checks immediately that $\nabla_{\dot{\gamma}}^{g} V=\dot{\gamma} \times V$, that is, the vector $V$ is parallel transported according to a helicoidal movement. If we now transport the vector along the edges of a closed triangle, it will be rotated around three linearly independent axes, hence the holonomy algebra is $\mathfrak{g}_{\mathrm{T}}^{*}=$ $\mathfrak{h}_{\mathrm{T}}^{*}=\mathfrak{s o}(3)$.

Example 4.2. Any 3 -form in $\mathbb{R}^{4}$ is equivalent to one of the forms $\mathrm{T}=a \cdot e_{123}$, hence the same argument as in the previous example yields that $\mathfrak{g}_{\mathrm{T}}^{*}=0$ or $\mathfrak{s o}(3)$.

Example 4.3. Any 3 -form in $\mathbb{R}^{5}$ is equivalent to one of the forms $\mathrm{T}=a \cdot e_{123}+b \cdot e_{345}$. The corresponding algebras are $\mathfrak{g}_{\mathrm{T}}=\mathfrak{s o}(5), \mathfrak{s o}(3) \oplus \mathfrak{s o}(2), 0$ and $\mathfrak{g}_{\mathrm{T}}^{*}=0, \mathfrak{s o}(3), \mathfrak{s o}(5)$.

Example 4.4. In $\mathbb{R}^{7}$, we consider the 3 -form $\mathrm{T}=e_{127}+e_{135}-e_{146}-e_{236}-e_{245}+e_{347}+e_{567}$. Its isotropy algebra $\mathfrak{g}_{\mathrm{T}}$ is isomorphic to the exceptional Lie algebra $\mathfrak{g}_{2}$. Moreover, $\mathfrak{s o}(7)$ splits into two $\mathrm{G}_{2}$-irreducible components, $\mathfrak{s o}(7)=\mathfrak{g}_{\mathrm{T}} \oplus \mathfrak{m}$. The orthogonal complement $\mathfrak{m}$ of $\mathfrak{g}_{\mathrm{T}}$ coincides with the space of all inner products $\left.X\right\lrcorner \mathrm{T}$. The Lie algebra generated by these elements is isomorphic to $\mathfrak{s o}(7)$. To summarize, we obtain $\mathfrak{g}_{\mathrm{T}}=\mathfrak{g}_{2}$ and $\mathfrak{g}_{\mathrm{T}}^{*}=\mathfrak{s o}(7)$.

The first Proposition estimates the dimension of the Lie algebra $\mathfrak{g}_{\mathrm{T}}^{*}$ from below.

Proposition 4.1. Let $\mathrm{T} \in \Lambda^{3}\left(\mathbb{R}^{n}\right)$ be a 3 -form and $\Phi_{\mathrm{T}}: \mathbb{R}^{n} \rightarrow \mathfrak{g}_{\mathrm{T}}^{*}$ be the map defined by the formula $\left.\Phi_{\mathrm{T}}(X):=X\right\lrcorner \mathrm{T}$. Then $\mathrm{T}$ depends only on the orthogonal complement $\operatorname{Ker}\left(\Phi_{\mathrm{T}}\right)^{\perp}$,

$$
\mathrm{T} \in \Lambda^{3}\left(\operatorname{Ker}\left(\Phi_{\mathrm{T}}\right)^{\perp}\right)
$$

In particular, if $\mathrm{T}$ is a 3-form which can not be reduced to a lower dimensional subspace, then

$$
n \leq \operatorname{dim}\left(\mathfrak{g}_{\mathrm{T}}^{*}\right)
$$

Next, we investigate the representation of the Lie algebra $\mathfrak{g}_{\mathrm{T}}^{*}$ in $\mathbb{R}^{n}$.

Proposition 4.2. The representation $\left(\mathfrak{g}_{\mathrm{T}}^{*}, \mathbb{R}^{n}\right)$ is reducible if and only if there exist a proper subspace $\mathrm{V} \subset \mathbb{R}^{n}$ and two 3 -forms $\mathrm{T}_{1} \in \Lambda^{3}(\mathrm{~V})$ and $\mathrm{T}_{2} \in \Lambda^{3}\left(\mathrm{~V}^{\perp}\right)$ such that $\mathrm{T}=\mathrm{T}_{1}+\mathrm{T}_{2}$. In this case, the Lie algebra $\mathfrak{g}_{\mathrm{T}}^{*}$ decomposes into

$$
\mathfrak{g}_{\mathrm{T}}^{*}=\mathfrak{g}_{\mathrm{T}_{1}}^{*} \oplus \mathfrak{g}_{\mathrm{T}_{2}}^{*} \text {. }
$$

\footnotetext{
2 "Deux trièdres [...] de $\mathcal{E}$ seront parallèles lorsque les trièdres correspondants de $\mathrm{E}$ [l'espace euclidien classique] pourront se déduire l'un de l'autre par un déplacement hélicoïdal de pas donné, de sens donné[...]. L'espace $\mathcal{E}$ ainsi défini admet un groupe de transformations à 6 paramètres : ce serait notre espace ordinaire vu par des observateurs dont toutes les perceptions seraient tordues." loc.cit.
} 
Proof. Consider a $\mathfrak{g}_{\mathrm{T}}^{*}$-invariant subspace $\mathrm{V} \subset \mathbb{R}^{n}$ and fix a basis $e_{1}, \cdots, e_{k}$ in $\mathrm{V}$ as well as a basis $e_{k+1}, \cdots, e_{n}$ in its orthogonal complement $\mathrm{V}^{\perp}$. Then, for any vector $X \in \mathbb{R}^{n}$, and any pair of indices $1 \leq i \leq k, k+1 \leq \alpha \leq n$, we obtain

$$
\mathrm{T}\left(X, e_{i}, e_{\alpha}\right)=0 \text {. }
$$

Since $\mathrm{T}$ is skew-symmetric, we conclude

$$
\mathrm{T}\left(e_{i}, e_{j}, e_{\alpha}\right)=0, \quad \text { and } \mathrm{T}\left(e_{i}, e_{\alpha}, e_{\beta}\right)=0 .
$$

The following Proposition restricts the type of the Lie algebra $\mathfrak{g}_{\mathrm{T}}^{*}$. In particular, it cannot be contained in the Lie algebra $\mathfrak{u}(k) \subset \mathfrak{s o}(2 k)$ of the unitary group.

Proposition 4.3. Let $\mathrm{T}$ be a 3 -form in $\mathbb{R}^{2 k}$ and suppose that there exists a 2 -form $\Omega$ such that

Then $\mathrm{T}$ is zero, $\mathrm{T}=0$.

$$
\Omega^{k} \neq 0 \text { and }\left[\mathfrak{g}_{\mathrm{T}}^{*}, \Omega\right]=0 \text {. }
$$

Proof. We fix an orthonormal basis in $\mathbb{R}^{2 k}$ such that the 2 -form $\Omega$ is given by

$$
\Omega=A_{1} \cdot e_{12}+\cdots+A_{k} \cdot e_{2 k-1,2 k}, \quad A_{1} \cdot \ldots \cdot A_{k} \neq 0 .
$$

The condition $\left[\mathfrak{g}_{\mathrm{T}}^{*}, \Omega\right]=0$ is equivalent to the equations

$$
\sum_{j=1}^{2 k} \Omega_{\alpha j} \cdot \mathrm{T}_{\beta j \gamma}=\sum_{j=1}^{2 k} \mathrm{~T}_{\beta \alpha j} \cdot \Omega_{j \gamma}
$$

for any triple $1 \leq \alpha, \beta, \gamma \leq 2 k$. Using the special form of $\Omega$ we obtain the equations $(1 \leq \alpha, \gamma \leq k)$ :

$$
A_{\alpha} \cdot \mathrm{T}_{\beta, 2 \alpha, 2 \gamma-1}=-A_{\gamma} \cdot \mathrm{T}_{\beta, 2 \alpha-1,2 \gamma}
$$

and

$$
A_{\alpha} \cdot \mathrm{T}_{\beta, 2 \alpha-1,2 \gamma-1}=A_{\gamma} \cdot \mathrm{T}_{\beta, 2 \alpha, 2 \gamma} .
$$

The latter system of algebraic equations implies that $\mathrm{T}=0$ vanishes. Indeed, let us compute - for example $-\mathrm{T}_{\beta, 2 \alpha, 2 \gamma-1}$. In case $\beta$ is odd, we have

$$
\begin{aligned}
A_{\alpha} \cdot \mathrm{T}_{\beta, 2 \alpha, 2 \gamma-1} & =-A_{\gamma} \cdot \mathrm{T}_{\beta, 2 \alpha-1,2 \gamma}=A_{\gamma} \cdot \mathrm{T}_{2 \alpha-1, \beta, 2 \gamma}=-A_{(\beta+1) / 2} \cdot \mathrm{T}_{2 \gamma-1,2 \alpha-1, \beta+1} \\
& =A_{\alpha} \cdot \mathrm{T}_{2 \gamma-1,2 \alpha, \beta}=-A_{\alpha} \cdot \mathrm{T}_{\beta, 2 \alpha, 2 \gamma-1} .
\end{aligned}
$$

In case $\beta$ is even, a similar computations yields the formula

$$
\left[A_{\alpha}\right]^{2} \cdot \mathrm{T}_{\beta, 2 \alpha, 2 \gamma-1}=-\left[A_{\beta / 2}\right]^{2} \cdot \mathrm{T}_{\beta, 2 \alpha, 2 \gamma-1} .
$$

Theorem 4.1. For any 3 -form $\mathrm{T} \in \Lambda^{3}\left(\mathbb{R}^{n}\right)$, the Lie algebra $\mathfrak{g}_{\mathrm{T}}^{*}$ is semisimple and coincides with the holonomy algebra $\mathfrak{h}_{\mathrm{T}}^{*}$.

Proof. According to Proposition 4.2 we assume that the representation $\left(\mathfrak{g}_{\mathrm{T}}^{*}, \mathbb{R}^{n}\right)$ is irreducible. The Lie algebra $\mathfrak{g}_{\mathrm{T}}^{*}$ splits into the holonomy algebra $\mathfrak{h}_{\mathrm{T}}^{*}$ and the center $\mathfrak{z}\left(\mathfrak{g}_{\mathrm{T}}^{*}\right)$. Suppose that the center $\mathfrak{z}$ is non trivial, i.e., that there exist a 2 -form $\Omega$ such that

$$
\left[\mathfrak{g}_{\mathrm{T}}^{*}, \Omega\right]=0 \text {. }
$$

We split the euclidian space into

$$
\mathbb{R}^{n}=\operatorname{Ker}(\Omega) \oplus \operatorname{Ker}(\Omega)^{\perp}
$$

and observe that both subspaces are $\mathfrak{g}_{\mathrm{T}}^{*}$-invariant. Since $\operatorname{Ker}(\Omega) \neq 0$ and the representation $\left(\mathfrak{g}_{\mathrm{T}}^{*}, \mathbb{R}^{n}\right)$ is irreducible, we conclude that $\operatorname{Ker}(\Omega)=0$. In particular, the dimension $n=2 k$ is even and $\Omega^{k} \neq 0$. Finally, we obtain $\mathrm{T}=0$ by Proposition 4.3. 
A second restriction for the algebra $\mathfrak{g}_{\mathrm{T}}^{*}$ results from the observation that it is not contained in the isotropy Lie algebra of a spinor. This fact implies that there are no $\nabla^{\mathrm{T}}$-parallel spinors in $\mathbb{R}^{n}$ for $\mathrm{T} \neq 0$. Furthermore, certain semisimple Lie groups cannot occur as holonomy groups of 3 -form in $\mathbb{R}^{n}$. In dimensions $n \leq 9$, where the group $\operatorname{Spin}(n)$ acts transitively on the set of spinors of length one, the proof is a consequence of a direct algebraic computation. For example, in dimension $n=8$, a general 3 -form depends on 56 parameters and $\mathfrak{g}_{\mathrm{T}}^{*} \cdot \psi=0$ is a system consisting again of at least 56 linear equations. In higher dimensions, we have to avoid the problem of the unknown orbit structure of the spin representation. We use a global argument here, but it would be interesting to find a purely algebraic proof.

Theorem 4.2. Let $\mathrm{T} \in \Lambda^{3}\left(\mathbb{R}^{n}\right)$ be a 3-form. If there exists a non trivial spinor $\psi \in \Delta_{n}$ such that $\mathfrak{g}_{\mathrm{T}}^{*} \cdot \psi=0$, then $\mathrm{T}=0$.

Proof. Consider the compact, flat torus $\mathbb{R}^{n} / \mathbb{Z}^{n}$. Since $\mathrm{T}$ and $\psi \in \Delta_{n}$ are constant, both are geometric objects on the torus. In particular, with respect to the trivial spin structure of the torus, $\psi$ is a $\nabla^{\mathrm{T}}$-parallel spinor field on $\mathbb{R}^{n} / \mathbb{Z}^{n}$. The integral formula of Theorem 6.3 yields that $\mathrm{T}=0$.

Corollary 4.1. Let $\mathrm{T} \in \Lambda^{3}\left(\mathbb{R}^{n}\right)$ a 3-form. If there exists a non trivial solution $\psi$ : $\mathbb{R}^{n} \rightarrow \Delta_{n}$ of the equation

$$
\left.\nabla_{X}^{\mathrm{T}} \psi=X(\psi)+(X\lrcorner \mathrm{T}\right) \cdot \psi=0,
$$

then $\mathrm{T}=0$ and $\psi$ is constant.

Proof. Suppose that a non trivial parallel spinor exists. By Corollary 3.1 and Theorem 4.1. we conclude that $\psi$ is constant and $\mathfrak{g}_{\mathrm{T}}^{*} \cdot \psi=0$. Theorem 4.2 yields now that the 3 -form $\mathrm{T}=0$ vanishes.

In low dimensions, we obtain a complete list of all possible holonomy algebras $\mathfrak{h}_{\mathrm{T}}^{*}$ :

- $n=5: \quad \mathfrak{h}_{\mathrm{T}}^{*}=0, \mathfrak{s o}(3), \mathfrak{s o}(5)$.

- $n=6: \quad \mathfrak{h}_{\mathrm{T}}^{*}=0, \mathfrak{s o}(3), \mathfrak{s o}(5), \mathfrak{s o}(3) \oplus \mathfrak{s o}(3), \mathfrak{s o}(6)$.

- $n=7: \quad \mathfrak{h}_{\mathrm{T}}^{*}=0, \mathfrak{s o}(3), \mathfrak{s o}(5), \mathfrak{s o}(3) \oplus \mathfrak{s o}(3), \mathfrak{s o}(6), \mathfrak{s o}(7)$.

Starting from dimension eight, there occur representations of all semisimple Lie algebras as the holonomy algebra of certain 3-form in euclidian space. Indeed, suppose that the euclidian space $\mathbb{R}^{n}=\mathfrak{g}$ is a compact Lie algebra, and the inner product and the Lie bracket are related by the condition

$$
\langle[X, Y], Z\rangle+\langle Y,[X, Z]\rangle=0 .
$$

Then $\mathrm{T}(X, Y, Z):=\langle[X, Y], Z\rangle$ is a 3 -form in $\mathbb{R}^{n}=\mathfrak{g}$ and we obtain

$$
X\lrcorner \mathrm{T}=\operatorname{ad}(X) \in \mathfrak{s o}(\mathfrak{g})=\mathfrak{s o}(n) .
$$

The Lie algebra $\mathfrak{g}_{T}^{*}$ is the image of the Lie algebra $\mathfrak{g}$ under the adjoint representation. Consequently, we have a series of representations occurring for some 3 -form.

Proposition 4.4. The adjoint representation of any compact, semisimple Lie algebra $\mathfrak{g}$ is the holonomy algebra of a certain 3-form with constant coefficients in euclidian space $\mathfrak{g}=\mathbb{R}^{n}$.

The first interesting example is the 8-dimensional Lie algebra $\mathfrak{s u}(3)$. It yields a 3 -form in $\mathbb{R}^{8}$ such that $\mathfrak{g}_{\mathrm{T}}^{*}=\mathfrak{s u}(3)$ and the inclusion $\mathfrak{s u}(3) \subset \mathfrak{s o}(8)$ is the adjoint representation. This example realizes the lower bound in the dimension estimate of Proposition 4.1. 
The latter series of examples generalizes to Riemannian naturally reductive spaces $G / H$. Decompose the Lie algebra

$$
\mathfrak{g}=\mathfrak{h} \oplus \mathfrak{m}, \quad \operatorname{Ad}(H)(\mathfrak{m}) \subset \mathfrak{m},
$$

and consider the canonical connection of the reductive space. Its torsion form is given by the formula

$$
\mathrm{T}(X, Y, Z)=-\left\langle[X, Y]_{\mathfrak{m}}, Z\right\rangle, \quad X, Y, Z \in \mathfrak{m},
$$

where $[,]_{\mathfrak{m}}$ denotes the $\mathfrak{m}$-part of the Lie bracket. Consider the euclidian space $\mathfrak{m}$ and the 3 -form T. Then $\mathfrak{g}_{\mathrm{T}}^{*}$ is the Lie subalgebra of $\mathfrak{s o}(\mathfrak{m})$ generated by the subspace $\mathfrak{m} \rightarrow \mathfrak{s o}(\mathfrak{m})$, where this map is given by the formula

$$
Z \longrightarrow Z\lrcorner \mathrm{T}, \quad(Z\lrcorner \mathrm{T})(X)=[X, Z]_{\mathfrak{m}}, \quad Z \in \mathfrak{m} .
$$

In general, this is not the isotropy representation of the reductive space, but related to the holonomy of its Levi-Civita connection (see [21]).

Let us discuss the question which irreducible representations $\left(\mathfrak{g}, \mathbb{R}^{n}\right)$ of a semisimple Lie algebra $\mathfrak{g}$ can occur for a 3 -form. We already know some restrictions. In even dimensions, the $\mathfrak{g}$-action cannot preserve a non-degenerate 2 -form and, in any dimension, the lift into the spin representation cannot preserve a spinor. In order to formulate a further restriction we introduce - in analogy to the prolongation of a linear Lie algebra (see [21, note 13]) - an antisymmetric prolongation of a representation of a compact semisimple Lie algebra by

$$
\left.\mathrm{T}\left(\mathfrak{g}, \mathbb{R}^{n}\right):=\left\{\mathrm{T} \in \Lambda^{3}\left(\mathbb{R}^{n}\right): X\right\lrcorner \mathrm{T} \in \mathfrak{g} \text { for any } X \in \mathbb{R}^{n}\right\} .
$$

The subspace $\mathrm{T}\left(\mathfrak{g}, \mathbb{R}^{n}\right) \subset \Lambda^{3}\left(\mathbb{R}^{n}\right)$ is $\mathfrak{g}$-invariant. A 3 -form $\mathrm{T}$ belongs to this space if and only if its Lie algebra is contained in $\mathfrak{g}_{\mathrm{T}}^{*} \subset \mathfrak{g}$. In particular, we can formulate a necessary condition.

Proposition 4.5. If a representation $\left(\mathfrak{g}, \mathbb{R}^{n}\right)$ of a compact, semisimple Lie algebra is realized by some 3 -form $\mathrm{T} \in \Lambda^{3}\left(\mathbb{R}^{n}\right)$, then $\mathrm{T}\left(\mathfrak{g}, \mathbb{R}^{n}\right) \neq 0$ is non trivial.

Example 4.5. The unique irreducible 16-dimensional representation $\mathfrak{s p i n}(9) \subset \mathfrak{s o}(16)$ of the Lie algebra $\mathfrak{s p i n}(9)$ does not admit invariant, non degenerate 2 -forms in $\mathbb{R}^{16}$ or invariant spinors in $\Delta_{16}$. This algebra satisfies the conditions of Proposition 4.3 and Theorem 4.2 However, the algebra and any non trivial subalgebra of it cannot be the algebra $\mathfrak{g}_{\mathrm{T}}^{*}$ for a 3 -form $\mathrm{T}$ in sixteen variables. It turns out that

$$
\mathrm{T}\left(\mathfrak{s p i n}(9), \mathbb{R}^{16}\right)=0 .
$$

The proof is a longer algebraic computation and will be postponed to the appendix.

We remark that the results of this section cannot be generalized directly to the case of $k$-forms. Spinorial connections related with forms of higher degree behave differently. Theorem 4.2 and Corollary 4.1 are not true for 4-forms. Especially interesting is dimension eight. A 4-form $\mathrm{T}$ on $\mathbb{R}^{8}$ depends on 70 parameters. On the other hand, the 8-dimensional spin representation is real and splits $\Delta_{8}=\Delta_{8}^{+} \oplus \Delta_{8}^{-}$into two 8-dimensional representations. Consider a spinor $\psi \in \Delta_{8}^{+}$in one of these components. The Clifford product $(X\lrcorner \mathrm{T}) \cdot \psi$ is a spinor in $\Delta_{8}^{-}$and the condition $\left.(X\lrcorner \mathrm{T}\right) \cdot \psi=0$ for any $X \in \mathbb{R}^{8}$ is a system of $8 \cdot 8=64$ linear equations for the coefficients of the 4 -form T. Consequently, any spinor $\psi \in \Delta_{8}^{ \pm}$admits a family of 4 -forms $\mathrm{T}$ depending at least on 6 parameters such that $\mathfrak{g}_{\mathrm{T}}^{*} \cdot \psi=0$. In fact, the number of parameters is seven. Indeed, for any spinor $\psi$, we consider the subspace $\left\{\mathrm{T} \in \Lambda^{k}\left(\mathbb{R}^{n}\right): \mathfrak{g}_{\mathrm{T}}^{*} \cdot \psi=0\right\}$. It is invariant under the isotropy group of the spinor. In dimension eight, the isotropy group $\operatorname{Spin}(7)$ splits $\Lambda^{4}\left(\mathbb{R}^{8}\right)$ into 
four $\operatorname{Spin}(7)$-irreducible components of dimensions 1, 7, 27, 35 (see [10]). In any case, there exist non trivial 4 -forms on $\mathbb{R}^{8}$ with non trivial parallel spinors. Since the space $\Delta_{8}$ of all spinors in dimension eight coincides with the space $\Delta_{9}$ of all spinors in dimension nine, we obtain 4 -forms in $\mathbb{R}^{9}$ with parallel spinors, too.

\section{5. $\nabla$-parallel 2-forms on manifolds}

Any metric connection on a Riemannian manifold defines several differential operators, like the Laplace operator on forms or the Dirac operator on spinors. One can compare these operators with the corresponding operator defined by the Levi-Civita connection. There is one particularly interesting formula of that type, namely for the codifferential of an exterior form,

$$
\left.\delta^{\nabla} \omega:=-\sum_{i=1}^{n} e_{i}\right\lrcorner \nabla_{e_{i}} \omega .
$$

We shall prove that the Riemannian divergence of the torsion form coincides with its $\nabla$-divergence.

Proposition 5.1. Let $\nabla$ be a connection with skew-symmetric torsion. Then, for any exterior form $\omega$, the following formula holds:

$$
\left.\left.\left.\left.\delta^{\nabla} \omega=\delta^{g} \omega-\frac{1}{2} \cdot \sum_{i, j=1}^{n}\left(e_{i}\right\lrcorner e_{j}\right\lrcorner \mathrm{~T}\right) \wedge\left(e_{i}\right\lrcorner e_{j}\right\lrcorner \omega\right) .
$$

In particular, for the torsion form itself, we obtain $\delta^{\nabla} \mathrm{T}=\delta^{g} \mathrm{~T}$.

Proof. For simplicity, we prove the formula for 3-forms. Then we get

$$
\begin{aligned}
\delta^{\nabla} \omega(X, Y)= & -\sum_{i=1}^{n} \nabla_{e_{i}} \omega\left(e_{i}, X, Y\right)=-\sum_{i=1}^{n} e_{i}\left(\omega\left(e_{i}, X, Y\right)\right) \\
& +\sum_{i=1}^{n}\left[\omega\left(\nabla_{e_{i}} e_{i}, X, Y\right)+\omega\left(e_{i}, \nabla_{e_{i}} X, Y\right)+\omega\left(e_{i}, X, \nabla_{e_{i}} Y\right)\right] .
\end{aligned}
$$

Since the two connections are related by $2 \cdot \nabla_{X} Y-2 \cdot \nabla_{X}^{g} Y=\sum_{j=1}^{n} \mathrm{~T}\left(X, Y, e_{j}\right) \cdot e_{j}$, this can be rewritten in the form

$$
\begin{aligned}
\delta^{\nabla} \omega(X, Y) & =\delta^{g} \omega(X, Y)+\frac{1}{2} \sum_{i, j=1}^{n}\left[\mathrm{~T}\left(e_{i}, X, e_{j}\right) \omega\left(e_{i}, e_{j}, Y\right)+\mathrm{T}\left(e_{i}, Y, e_{j}\right) \omega\left(e_{i}, X, e_{j}\right)\right] \\
& \left.\left.\left.\left.=\delta^{g} \omega(X, Y)-\frac{1}{2} \sum_{i, j=1}^{n}\left(e_{i}\right\lrcorner e_{j}\right\lrcorner \mathrm{~T}\right) \wedge\left(e_{i}\right\lrcorner e_{j}\right\lrcorner \omega\right)(X, Y) .
\end{aligned}
$$

Corollary 5.1. If the torsion form $\mathrm{T}$ is $\nabla$-parallel, then its divergence vanishes,

$$
\delta^{g} \mathrm{~T}=\delta^{\nabla} \mathrm{T}=0 .
$$

Let us discuss $\nabla$-parallel 2-forms. The differential equation reads as

$$
\nabla_{\beta}^{g} \Omega_{\alpha \gamma}=\frac{1}{2} \sum_{j=1}^{n}\left\{\Omega_{\gamma j} \cdot \mathrm{T}_{\beta j \alpha}-\Omega_{\alpha j} \cdot \mathrm{T}_{\beta j \gamma}\right\} .
$$

Using the well known formulas for the exterior differential, the codifferential as well as for the Bochner-Laplace operator $\nabla^{*} \nabla$ we obtain 
Proposition 5.2. Let $\nabla$ be a metric connection $\nabla$ and skew-symmetric torsion. If $\Omega$ is a $\nabla$-parallel 2-form, then

$$
\begin{aligned}
\delta^{g} \Omega & \left.=\frac{1}{2} \cdot(\Omega\lrcorner \mathrm{T}\right)=\frac{1}{4} \sum_{j, \beta, \gamma=1}^{n} \Omega_{\beta j} \cdot \mathrm{T}_{\beta j \gamma} \cdot e_{\gamma}, \\
d \Omega & \left.\left.=\sum_{j=1}^{n}\left(e_{j}\right\lrcorner \Omega\right) \wedge\left(e_{j}\right\lrcorner \mathrm{T}\right) \\
& =\frac{1}{6} \sum_{j, \alpha, \beta, \gamma=1}^{n}\left\{\Omega_{\alpha j} \cdot \mathrm{T}_{\beta j \gamma}-\Omega_{\beta j} \cdot \mathrm{T}_{\alpha j \gamma}+\Omega_{\gamma j} \cdot \mathrm{T}_{\alpha j \beta}\right\} \cdot e_{\alpha} \wedge e_{\beta} \wedge e_{\gamma}, \\
g\left(\Omega, \nabla^{*} \nabla^{g} \Omega\right) & =\frac{1}{2} \sum_{j, k, \alpha, \beta, \gamma=1}^{n} \Omega_{\alpha \gamma} \cdot \Omega_{\alpha k} \cdot \mathrm{T}_{\beta j k} \cdot \mathrm{T}_{\beta j \gamma},
\end{aligned}
$$

where $\nabla^{*} \nabla^{g}$ denotes the Riemannian Bochner-Laplace operator acting on 2-forms.

In an adapted basis, $\Omega=A_{1} \cdot e_{1} \wedge e_{2}+\cdots+A_{k} \cdot e_{2 k-1} \wedge e_{2 k}$, the third formula simplifies,

$$
g\left(\Omega, \nabla^{*} \nabla^{g} \Omega\right)=\frac{1}{2} \sum_{\kappa=1}^{k} \sum_{i, j=1}^{n}\left(\mathrm{~T}_{i j 2 \kappa-1}^{2}+\mathrm{T}_{i j 2 \kappa}^{2}\right) \cdot A_{\kappa}^{2} .
$$

It explains once again, from a geometric point of view, the proof of Proposition 4.3 We remark that there exist indeed metric connections with skew-symmetric torsion and parallel 2-forms. Indeed, consider an almost hermitian manifold with totally skewsymmetric Nijenhuis tensor. Then there is a unique connection $\nabla$ preserving the hermitian structure with skew-symmetric torsion (see [15]). The fundamental form of the hermitian structure is $\nabla$-parallel. A second example are Sasakian manifolds. For these, the differential of the contact form is parallel with respect to the unique connection preserving the Sasakian structure.

\section{Schrödinger-Lichnerowicz type formulas for Dirac operators}

Consider a Riemannian spin manifold $\left(M^{n}, g, \mathrm{~T}\right)$ with 3 -form $\mathrm{T}$ as well as the oneparameter family of linear metric connections with torsion,

$$
\nabla_{X}^{s} Y:=\nabla_{X}^{g} Y+2 s \cdot \mathrm{T}(X, Y,-) .
$$

In particular, the superscript $s=0$ corresponds to the Levi-Civita connection, $\nabla^{g} \equiv \nabla^{0}$. These connections can all be lifted to connections on the spinor bundle $S$ of $M$, where they take the expression

$$
\left.\nabla_{X}^{s} \psi:=\nabla_{X}^{g} \psi+s(X\lrcorner \mathrm{T}\right) \cdot \psi .
$$

There is a formula for the square of the Dirac operator $D^{s}$ associated with the connection $\nabla^{s}$. In order to state it, let us introduce the first order differential operator

$$
\left.\left.\left.\mathcal{D}^{s} \psi:=\sum_{k=1}^{n}\left(e_{k}\right\lrcorner \mathrm{T}\right) \cdot \nabla_{e_{k}}^{s} \psi=\mathcal{D}^{0} \psi+s \sum_{k}\left(e_{k}\right\lrcorner \mathrm{T}\right) \cdot\left(e_{k}\right\lrcorner \mathrm{T}\right) \cdot \psi,
$$

where $e_{1}, \ldots, e_{n}$ denotes an orthonormal basis. In fact, it will be convenient to use a separate notation for the algebraic 4-form derived from $\mathrm{T}$ appearing in the difference 
$\mathcal{D}^{s}-\mathcal{D}^{0}$

$$
\left.\left.\sigma_{\mathrm{T}}:=\frac{1}{2} \sum_{k}\left(e_{k}\right\lrcorner \mathrm{T}\right) \wedge\left(e_{k}\right\lrcorner \mathrm{T}\right) .
$$

Theorem 6.1 ([15, Thm 3.1, 3.3]). Let $\left(M^{n}, g, \nabla^{s}\right)$ be an n-dimensional Riemannian manifold with a metric connection $\nabla^{s}$ of skew-symmetric torsion $4 \cdot s \cdot \mathrm{T}$. Then, the square of the Dirac operator $D^{s}$ associated with $\nabla^{s}$ acts on an arbitrary spinor field $\psi$ as

$$
\left(D^{s}\right)^{2} \psi=\Delta^{s}(\psi)+3 s d \mathrm{~T} \cdot \psi-8 s^{2} \sigma_{\mathrm{T}} \cdot \psi+2 s \delta \mathrm{T} \cdot \psi-4 s \mathcal{D}^{s} \psi+\frac{1}{4} \mathrm{Scal}^{s} \cdot \psi,
$$

where $\Delta^{s}$ is the spinor Laplacian of $\nabla^{s}$,

$$
\Delta^{s}(\psi)=\left(\nabla^{s}\right)^{*} \nabla^{s} \psi=-\sum_{k=1}^{n} \nabla_{e_{k}}^{s} \nabla_{e_{k}}^{s} \psi+\nabla_{\nabla_{e_{i}}^{g} e_{i}}^{s} \psi .
$$

Furthermore, the anticommutator of $D^{s}$ and $\omega$ is

$$
D^{s} \cdot \mathrm{T}+\mathrm{T} \cdot D^{s}=d \mathrm{~T}+\delta \mathrm{T}-8 s \cdot \sigma_{\mathrm{T}}-2 \mathcal{D}^{s} .
$$

$\mathrm{Scal}^{s}$ denotes the scalar curvature of the connection $\nabla^{s}$. Remark that Scal ${ }^{0}=\mathrm{Scal}^{g}$ is the usual scalar curvature of the underlying Riemannian manifold $\left(M^{n}, g\right)$.

This formula for $\left(D^{s}\right)^{2}$ has the disadvantage of still containing a first order differential operator as well as several 4-forms, which are difficult to treat algebraically. Inspired by the homogeneous case, we were looking for an alternative comparison of $\left(D^{s}\right)^{2}$ with the Laplace operator of some other connection $\nabla^{s^{\prime}}$ from the same family. For the computations, we need the square of $\mathrm{T}$ inside the Clifford algebra. The proof of the following proposition is completely similar to that of Proposition 3.1 in [1] and will hence be omitted.

Proposition 6.1. Let $\mathrm{T}$ be a 3-form, and denote by the same symbol its associated $(2,1)$-tensor. Then its square inside the Clifford algebra has no contribution of degree 6 , and its scalar and fourth degree part are given by

$$
\mathrm{T}_{0}^{2}=\frac{1}{6} \sum_{i, j=1}^{n}\left\|\mathrm{~T}\left(e_{i}, e_{j}\right)\right\|^{2}, \quad \mathrm{~T}_{4}^{2}=-2 \cdot \sigma_{\mathrm{T}} .
$$

With these preparations in hand, we can state a more useful Schrödinger-Lichnerowicz type formula for $\left(D^{s}\right)^{2}$. It links the Dirac operator for the parameter $s / 3$ with the Laplacian for the parameter $s$. The remainder is a zero order operator. Similar formulas can be found in [3] and, for homogeneous spaces, in [1].

Theorem 6.2. The spinor Laplacian $\Delta^{s}$ and the square of the Dirac operator $D^{s / 3}$ are related by

$$
\left(D^{s / 3}\right)^{2}=\Delta^{s}+s \cdot d \mathrm{~T}+\frac{1}{4} \mathrm{Scal}^{g}-2 s^{2} \cdot \mathrm{T}_{0}^{2} .
$$

Proof. By the formula from Theorem 6.1.

$$
\left(D^{s}\right)^{2}+4 s \mathcal{D}^{s}=\Delta^{s}+3 s d \mathrm{~T}-8 s^{2} \sigma_{\mathrm{T}}+2 s \delta \mathrm{T}+\frac{1}{4} \mathrm{Scal}^{s} .
$$

But since $D^{s}=D^{0}+3 s \cdot \mathrm{T}$, the left hand side can equally be rewritten

$$
\left(D^{s}\right)^{2}+4 s \mathcal{D}^{s}=\left(D^{0}\right)^{2}+3 s\left(\mathrm{~T} D^{0}+D^{0} \mathrm{~T}\right)+9 s^{2} \mathrm{~T}^{2}+4 s \mathcal{D}^{s} .
$$


We use equation (2) to express $\mathcal{D}^{s}$ by the anticommutator $\mathrm{T} D^{s}+D^{s} \mathrm{~T}$ :

$$
2 \mathcal{D}^{s}=d \mathrm{~T}+\delta \mathrm{T}-8 s \cdot \sigma_{\mathrm{T}}-\left(D^{0} \mathrm{~T}+\mathrm{T} D^{0}\right)-6 s \cdot \mathrm{T}^{2} .
$$

Now we obtain

$$
\begin{aligned}
\left(D^{s}\right)^{2}+4 s \mathcal{D}^{s} & =\left(D^{0}\right)^{2}+s\left(\mathrm{~T} D^{0}+D^{0} \mathrm{~T}\right)-3 s^{2} \mathrm{~T}^{2}-16 s^{2} \cdot \sigma_{\mathrm{T}}+2 s \cdot d \mathrm{~T} \\
& =\left(D^{s / 3}\right)^{2}-4 s^{2} \cdot \mathrm{T}^{2}-16 s^{2} \cdot \sigma_{\mathrm{T}}+2 s \cdot d \mathrm{~T} .
\end{aligned}
$$

We observe that $D^{s / 3}$ hence appears by quadratic completion. Now it suffices to insert this result in the formula of Theorem 6.1 and to use Proposition 6.1 as well as well as the easy relation between scalar curvatures, $\mathrm{Scal}^{s}=\mathrm{Scal}^{g}-24 s^{2} \mathrm{~T}_{0}^{2}$.

Integrating the latter formula on a compact manifold $M^{n}$, we obtain

$$
\int_{M^{n}}\left\|D^{s / 3} \psi\right\|^{2}=\int_{M^{n}}\left[\left\|\nabla^{s} \psi\right\|^{2}+s\langle d \mathrm{~T} \cdot \psi, \psi\rangle+\frac{1}{4} \mathrm{Scal}^{g} \cdot\|\psi\|^{2}-2 s^{2} \mathrm{~T}_{0}^{2} \cdot\|\psi\|^{2}\right] .
$$

A first consequence is a non linear version of Corollary 4.1 .

Theorem 6.3. Let $\left(M^{n}, g, \mathrm{~T}\right)$ be a compact, Riemannian spin manifold of non positive scalar curvature, $\mathrm{Scal}^{g} \leq 0$, and suppose that the 4 -form $d \mathrm{~T}$ acts on spinors as a non positive endomorphism. If there exists a solution $\psi \neq 0$ of the equation

$$
\left.\nabla_{X}^{\mathrm{T}} \psi=\nabla_{X}^{g} \psi+(X\lrcorner \mathrm{T}\right) \cdot \psi=0,
$$

then the 3 -form and the scalar curvature vanish, $\mathrm{T}=0=\mathrm{Scal}^{g}$, and $\psi$ is parallel with respect to the Levi-Civita connection.

Remark 6.1. Let us compare Theorem 6.3 with the integral formula in [15]. There, we need the condition that $d \mathrm{~T}+8 \cdot \sigma_{\mathrm{T}}$ is a non positive endomorphism in order to prove the same result. Since $\sigma_{\mathrm{T}}$ is neither positive nor negative, the two conditions are independent. The advantage of Theorem 6.3 is that only the algebraic type of the exterior differential $d \mathrm{~T}$ is involved, but not the algebraic type of the torsion form $\mathrm{T}$ itself (see the proof of Theorem 4.2).

Theorem 6.3 applies, in particular, to Calabi-Yau or Joyce manifolds. These are compact, Ricci-flat Riemannian manifolds in dimensions $n=6,7$ with one parallel spinor field. Let us perturb the connection $\nabla^{g}$ by a 3 -form such that $d \mathrm{~T}$ is non positive on spinors. Then the new connection $\nabla^{\mathrm{T}}$ does not admit $\nabla^{\mathrm{T}}$-parallel spinor fields. Nilmanifolds and their compact quotients $M^{n}=\mathrm{G} / \Gamma$ are a second family of examples where the theorem applies. A further family of examples arises from certain naturally reductive spaces and a torsion form $\mathrm{T}$ being proportional to the torsion form of the canonical connection, see [1.

\section{1-parameter families of connections with parallel spinors}

Consider a triple $\left(M^{n}, g, \mathrm{~T}\right)$ consisting of a Riemannian manifold together with a fixed 3 -form $\mathrm{T} \neq 0$. Let us ask for parameters $s_{0}$ such that the connection $\nabla^{s_{0}}$ admits a parallel spinor. The first example describes a case with parallel spinors for more then only one parameter in the family.

Example 7.1. Let $G$ be a simply connected Lie group, $g$ a biinvariant metric and consider the torsion form $\mathrm{T}(X, Y, Z):=g([X, Y], Z)$. The connections $\nabla^{ \pm 1 / 4}$ are flat (see [21]). In particular, there are $\nabla^{ \pm 1 / 4}$-parallel spinor fields. 
The integrability condition of Theorem 6.1 implies that the function

$$
\mathrm{G}(m, s):=\operatorname{Det}\left\{3 s d \mathrm{~T}-8 s^{2} \sigma_{\mathrm{T}}+2 s \delta \mathrm{T}+\frac{1}{4} \mathrm{Scal}^{s}\right\}(m)
$$

vanishes at $s_{0}$ and all $m \in M^{n}$. Here we treat forms as endomorphisms acting on spinors. The function $\mathrm{G}(s)$ is a polynomial. If the Riemannian scalar curvature is not identically zero, there exists only a finite number of parameters with $\nabla^{s}$-parallel spinors. Let us discuss low dimensions.

Example 7.2 (The 3-dimensional case). Consider the 3 -dimensional sphere $\left(S^{3}, g, d S^{3}\right)$ equipped with its standard metric and the volume form $\mathrm{T}=d S^{3}$. The equation

$$
\left.\nabla_{X}^{s} \psi=\nabla_{X}^{g} \psi+s \cdot(X\lrcorner \mathrm{T}\right) \cdot \psi=\nabla_{X}^{g} \psi+s \cdot X \cdot \psi=0
$$

is the usual Killing spinor equation. There are solutions on the 3-dimensional sphere for both parameters $s= \pm 1 / 2$. In dimension $n=3$, this is the only manifold admitting parallel spinors with respect to a non trivial 3 -form. Indeed, any $\mathrm{T}$ is proportional to the volume form, $\mathrm{T}=f \cdot d M^{3}$, where $f$ is a real-valued smooth function on $M^{3}$. If the equation

$$
\left.\nabla_{X}^{g} \psi+(X\lrcorner \mathrm{T}\right) \cdot \psi=\nabla_{X}^{g} \psi+f \cdot X \cdot \psi=0
$$

admits a non trivial solution $\psi$, then by a Theorem of A. Lichnerowicz (see [22]) $f$ is constant and $\left(M^{3}, g\right)$ is a space form.

In dimension four, we split any 2-form $\omega \in \Lambda^{2}\left(\mathbb{R}^{4}\right)$ into its self-dual and anti-self-dual part, $\omega=\omega_{+}+\omega_{-}$.

Lemma 7.1. An element $a+\omega+f \cdot e_{1234} \in \mathrm{Cl}\left(\mathbb{R}^{4}\right)$ acts on the space $\Delta_{4}$ of spinors and its determinant is given by the formula

$$
\operatorname{Det}\left[a+\omega+f \cdot e_{1234}\right]=\left[(a+f)^{2}+4 \cdot\left\|\omega_{+}\right\|^{2}\right] \cdot\left[(a-f)^{2}+4 \cdot\left\|\omega_{-}\right\|^{2}\right] .
$$

For any 3 -form $\mathrm{T} \in \Lambda^{3}\left(\mathbb{R}^{4}\right)$ the corresponding 4-form $\sigma_{\mathrm{T}}$ vanishes, $\sigma_{\mathrm{T}}=0$.

Proof. Any 3-form in $\mathbb{R}^{4}$ is $\mathrm{SO}(4)$-equivalent to the form $a \cdot e_{1} \wedge e_{2} \wedge e_{3}$ containing only one summand. This normal form implies $\sigma_{\mathrm{T}}=0$ immediately. The formula for the determinant follows from a matrix representation of the Clifford algebra.

The equation $\mathrm{G}(m, s)=0$ yields the following condition not expressing the full integrability conditions for the existence of a parallel spinor.

Proposition 7.1. Let $\left(M^{4}, g, \mathrm{~T}\right)$ be a Riemannian spin manifold equipped with a 3-form $\mathrm{T}$. If the connection $\nabla^{s}$ admits a non trivial parallel spinor, the following equations hold at any point:

(1) $12 \cdot s \cdot d \mathrm{~T}= \pm\left(\mathrm{Scal}^{g}-24 \cdot s^{2} \cdot \mathrm{T}_{0}^{2}\right) \cdot d M^{4}$.

(2) $\delta(\mathrm{T})$ is a (anti)-self-dual 2-form.

Example 7.3. Using the unique 3-dimensional example $S^{3}$ and its Killing spinors, we obtain by $M^{4}:=S^{3} \times \mathbb{R}^{1}$ and $\mathrm{T}:=d S^{3}$ an example in dimension four. Indeed, the 3 -dimensional Killing spinors are $\nabla^{ \pm 1 / 2}$-parallel on $M^{4}$. They do not depend on the $\mathbb{R}^{1}$-coordinate.

The integrability condition restricts the admissible parameters via a polynomial equation involving the scalar curvature and the torsion form of the triple $\left(M^{n}, g, \mathrm{~T}\right)$. Globally, not all of these values are possible. 
Theorem 7.1. Let $\left(M^{n}, g, \mathrm{~T}\right)$ be a compact triple. For any $\nabla^{s}$-parallel spinor $\psi$, the following formula holds:

$$
64 \cdot s^{2} \int_{M^{n}}\left\langle\sigma_{\mathrm{T}} \cdot \psi, \psi\right\rangle+\int_{M^{n}} \mathrm{Scal}^{s}=0 .
$$

If the mean value of $\left\langle\sigma_{\mathrm{T}} \cdot \psi, \psi\right\rangle$ does not vanish, the parameter $s$ is given by

$$
s=\frac{1}{8} \int_{M^{n}}\langle d \mathrm{~T} \cdot \psi, \psi\rangle / \int_{M^{n}}\left\langle\sigma_{\mathrm{T}} \cdot \psi, \psi\right\rangle
$$

If the mean value of $\left\langle\sigma_{\mathrm{T}} \cdot \psi, \psi\right\rangle$ vanishes, the parameter $s$ depends only on the Riemannian scalar curvature and on the length of the torsion form,

$$
0=\int_{M^{n}} \mathrm{Scal}^{s}=\int_{M^{n}} \mathrm{Scal}^{g}-24 s^{2} \int_{M^{n}} \mathrm{~T}_{0}^{2} .
$$

Finally, if the 4-forms $d \mathrm{~T}$ and $\sigma_{\mathrm{T}}$ are proportional, there are at most three parameters with $\nabla^{s}$-parallel spinors.

Proof. We use the integrability conditions for parallel spinors from Theorem 6.1] Let $\psi$ be a $\nabla^{s}$-parallel spinor of length one. Then we obtain

$$
3 s \int_{M^{n}}\langle d \mathrm{~T} \cdot \psi, \psi\rangle-8 s^{2} \int_{M^{n}}\left\langle\sigma_{\mathrm{T}} \cdot \psi, \psi\right\rangle+\frac{1}{4} \int_{M^{n}} \mathrm{Scal}^{g}-6 s^{2} \int_{M^{n}} \mathrm{~T}_{0}^{2}=0 .
$$

On the other side, the anticommutator relation between $D^{s}$ and $\mathrm{T}$ as well as the symmetry property of the Dirac operator in $\mathrm{L}^{2}$ yields

$0=\int_{M^{n}}\left\langle\mathrm{~T} \cdot \psi, D^{s} \psi\right\rangle=\int_{M^{n}}\left\langle D^{s} \mathrm{~T} \cdot \psi, \psi\right\rangle=\int_{M^{n}}\langle d \mathrm{~T} \cdot \psi, \psi\rangle-8 s \int_{M^{n}}\left\langle\sigma_{\mathrm{T}} \cdot \psi, \psi\right\rangle$.

If the mean values of $\left\langle\sigma_{\mathrm{T}} \cdot \psi, \psi\right\rangle$ does not vanish, then the second equation determines the parameter $s$,

$$
s=\frac{1}{8} \int_{M^{n}}\langle d \mathrm{~T} \cdot \psi, \psi\rangle / \int_{M^{n}}\left\langle\sigma_{\mathrm{T}} \cdot \psi, \psi\right\rangle .
$$

If the mean values of $\left\langle\sigma_{\mathrm{T}} \cdot \psi, \psi\right\rangle$ vanishes, then the mean value of $\langle d \mathrm{~T} \cdot \psi, \psi\rangle$ vanishes, too. The first formula yields the result.

Remark 7.1. In Proposition [8.5] we discuss an example of a non-flat connection on the compact, 7-dimensional Aloff-Wallach space $N(1,1)$ such that $\nabla^{s_{0}}$ and $\nabla^{-s_{0}}$ admit parallel spinors for suitable $s_{0}$, hence showing that both cases from Theorem [7.1] can actually occur in non-trivial situations. The "trivial" cases we knew about before are, of course, Lie groups (Example 17.1). Example 8.1 illustrates how a parallel spinor can occur for zero scalar curvature and $d \mathrm{~T}$ proportional to $\sigma_{\mathrm{T}}$. In the same vein, we construct on $N(1,1)$ a spinorial connection defined by a 4 -form $R$ such that $\nabla^{R}$ and $\nabla^{-R}$ admit parallel spinors (Proposition 8.13).

If the torsion form $\mathrm{T}$ of the linear connection is $\nabla$-parallel, we have $d \mathrm{~T}=2 \cdot \sigma_{\mathrm{T}}$ and $\delta(\mathrm{T})=0$. This situation occurs if $M^{n}=G / H$ is a reductive space and $\mathrm{T}$ is the torsion form of its natural connection (see [20]) or for Sasakian manifolds, nearly Kähler manifolds, nearly parallel $\mathrm{G}_{2}$-manifolds equipped with their unique connection preserving the corresponding geometric structure (see [15]). A direct consequence of Theorem 6.2 and Theorem 7.1 is the following 
Corollary 7.1. Let $\left(M^{n}, g, \mathrm{~T}\right)$ be a compact Riemannian manifold and suppose that the exterior differential of the 3-form $\mathrm{T}$ is proportional to the 4-form $\sigma_{\mathrm{T}}, d \mathrm{~T}=2 \cdot \sigma_{\mathrm{T}}$. If a connection $\nabla^{s}$ with $s \neq 1 / 4$ admits a parallel spinor field, the first eigenvalue of the Dirac operator $D^{s / 3}$ is bounded by

$$
6 \cdot \operatorname{vol}\left(M^{n}, g\right) \cdot \lambda_{1}^{2}\left(D^{s / 3}\right) \leq \int_{M^{n}} \mathrm{Scal}^{g} .
$$

If $\nabla^{1 / 4}=\nabla$ admits a parallel spinor field,

$$
\operatorname{vol}\left(M^{n}, g\right) \cdot \lambda_{1}^{2}\left(D^{1 / 12}\right) \leq \frac{1}{8} \int_{M^{n}} \mathrm{Scal}^{g}+\frac{1}{16} \int_{M^{n}} \mathrm{~T}_{0}^{2}
$$

Remark 7.2. On a naturally reductive space $M=G / K, \nabla^{1 / 4}$ is the canonical connection and [1, Corollary 3.1.] shows that a $\nabla^{1 / 4}$-parallel spinor realizes indeed this lower bound for $\lambda_{1}^{2}\left(D^{1 / 12}\right)$ provided the Casimir operator $\Omega_{\mathfrak{g}}$ is non-negative.

In case that the torsion form of the triple $\left(M^{n}, g, \mathrm{~T}\right)$ arises from some special nonintegrable geometric structure (see [14]), then usually only one connection in the family admits $\nabla^{s}$-parallel spinors. A uniqueness of that type requires additional arguments involving the special geometric structure. For example, consider a 5-dimensional Sasakian manifold $\left(M^{5}, g, \xi, \eta, \varphi\right)$. Denote by $\nabla$ its unique connection with skew-symmetric torsion and preserving the contact structure. Its torsion is given by the formula $\mathrm{T}=\eta \wedge d \eta$ (see [15]). In an adapted local frame, we have the formulas

$$
\mathrm{T}=\eta \wedge d \eta=2 \cdot\left(e_{12}+e_{34}\right) \wedge e_{5}, \quad d \eta=2 \cdot\left(e_{12}+e_{34}\right) .
$$

We consider the family $\nabla^{s}$ of connections. The first admissible case $s=1 / 4$ is the connection $\nabla$ preserving the contact structure we started with. In the papers [15, [16] the integrability conditions for $\nabla^{1 / 4}$-parallel spinors have been discussed completely. In particular, there are compact examples. For any Sasakian structure, we have

$$
\left.2 \cdot \nabla_{X}^{g} \xi=X\right\lrcorner d \eta \text {. }
$$

Suppose that there exists a $\nabla^{s}$-parallel spinor $\psi^{*}$ for some parameter $s \neq 1 / 4$. We introduce the vector field $\xi^{*}$ via the algebraic equation $\xi^{*} \cdot \psi^{*}=i \cdot \psi^{*}$. Then $\xi^{*}$ is $\nabla^{s}$-parallel,

$$
\nabla_{X}^{g} \xi^{*}=-2 s \cdot \mathrm{T}\left(X, \xi^{*},-\right) .
$$

Let us consider the inner product $f:=g\left(\xi, \xi^{*}\right)$ of the two vector fields. Its differential is given by the formula

$$
\left.2 \cdot d f=(4 s-1) \xi^{*}\right\lrcorner d \eta .
$$

In particular, $\xi(f)=\xi^{*}(f)=0$. Next, we compute the commutator of the vector fields

$$
\left.\left[\xi, \xi^{*}\right]=\nabla_{\xi}^{g} \xi^{*}-\nabla_{\xi^{*}}^{g} \xi=-(1 / 2+2 s) \xi^{*}\right\lrcorner d \eta=-\frac{4 s+1}{4 s-1} \cdot d f .
$$

First we discuss the case that $s \neq \pm 1 / 4$. Since $\left[\xi, \xi^{*}\right](f)=0$, we conclude that $\|\operatorname{grad}(f)\|^{2}=0$ holds and then we obtain $\left.\xi^{*}\right\lrcorner d \eta=0$. Consequently, $\xi^{*}$ is proportional to the vector field $\xi$. In particular, $\xi$ is $\nabla^{s}$-parallel,

$$
\left.\nabla_{X}^{g} \xi=-2 s \cdot \mathrm{T}(X, \xi,-)=2 s \cdot X\right\lrcorner d \eta .
$$

If $s \neq 1 / 4$, the latter equation contradicts the differential equation for the Killing vector field $\xi$ of a Sasakian structure. Finally, we study the remaining case $s=-1 / 4$. Then we have

$$
3 s \cdot d \mathrm{~T}-8 s^{2} \cdot \sigma_{\mathrm{T}}+\frac{1}{4} \cdot \mathrm{Scal}^{s}=-8 \cdot e_{1234}+\frac{1}{4} \cdot \mathrm{Scal}^{-1 / 4} .
$$


The endomorphism $e_{1234}$ acts on spinors with constant eigenvalues \pm 1 . Therefore, if $\psi^{*}$ is a $\nabla^{-1 / 4}$-parallel spinor, the scalar curvature $\mathrm{Scal}^{-1 / 4}$ is constant and $\psi^{*}$ is an eigenspinor of this endomorphism, $e_{1234} \cdot \psi^{*}=\epsilon \cdot \psi^{*}$. Since the connection $\nabla^{1 / 4}$ preserves the contact structure, the covariant derivative $\nabla_{X}^{1 / 4} \psi^{*}$ satisfies the same algebraic equation. With respect to

$$
\left.0=\nabla_{X}^{-1 / 4} \psi^{*}=\nabla_{X}^{1 / 4} \psi^{*}-\frac{1}{2} \cdot(X\lrcorner \mathrm{T}\right) \cdot \psi^{*}
$$

we conclude that for any vector $X$ the spinor $\psi^{*}$ satisfies the equation

$$
\left.\left.e_{1234} \cdot(X\lrcorner \mathrm{T}\right) \cdot \psi^{*}=\epsilon \cdot(X\lrcorner \mathrm{T}\right) \cdot \psi^{*} .
$$

Inserting $X=e_{1}$ we obtain $e_{1234} \cdot e_{25} \cdot \psi^{*}=\epsilon \cdot e_{25} \cdot \psi^{*}$ and $e_{1234} \cdot \psi^{*}=\epsilon \cdot \psi^{*}$. The relations in the Clifford algebra yield immediately that $\psi^{*}=0$. All together, we proved:

Proposition 7.2. Let $\left(M^{5}, g, \xi, \eta, \varphi\right)$ be a 5-dimensional Sasakian manifold and denote by $\nabla$ its unique connection with skew-symmetric torsion $\mathrm{T}$ and preserving the contact structure. If a connection $\nabla^{s}$ in the family through $\nabla$ admits a parallel spinor field, then $s=1 / 4$ and the connection is $\nabla$.

\section{Torsion forms with parallel spinors on Aloff-Wallach spaces}

The goal of this section is to construct on the Aloff-Wallach manifold $N(1,1)=\mathrm{SU}(3) / S^{1}$ a two-parameter family of metrics $g=g_{s, y}$ that admits, for every $g_{s, y}$, two inequivalent cocalibrated $\mathrm{G}_{2}$-structures. Moreover, we investigate the torsion forms of their unique connections (see [15]) as well as other geometric data of these connections. We use the computations available in [2, p.109 ff], which we hence shall not reproduce here. Consider the embedding $S^{1} \rightarrow \mathrm{SU}(3)$ given by $e^{i \theta} \mapsto \operatorname{diag}\left(e^{i \theta}, e^{i \theta}, e^{-2 i \theta}\right)$. The Lie algebra $\mathfrak{s u}(3)$ splits into $\mathfrak{s u}(3)=\mathfrak{m}+\mathbb{R}$, where $\mathbb{R}$ denotes the Lie algebra of $S^{1}$ deduced from the given embedding. The space $\mathfrak{m}$ has a preferred direction, namely the subspace $\mathfrak{m}_{0}$ generated by the matric $L:=\operatorname{diag}(3 i,-3 i, 0)$. Let $E_{i j}(i<j)$ be the matrix with 1 at the place $(i, j)$ and zero elsewhere, and define $A_{i j}=E_{i j}-E_{j i}, \tilde{A}_{i j}=i\left(E_{i j}+E_{j i}\right)$. We set $\mathfrak{m}_{1}:=\operatorname{Lin}\left\{A_{12}, \tilde{A}_{12}\right\}, \mathfrak{m}_{2}:=\operatorname{Lin}\left\{A_{13}, \tilde{A}_{13}\right\}$ and $\mathfrak{m}_{3}:=\operatorname{Lin}\left\{A_{23}, \tilde{A}_{23}\right\}$. The sum $\mathfrak{m}_{1} \oplus \mathfrak{m}_{2} \oplus \mathfrak{m}_{3}$ is an algebraic complement of $\mathfrak{m}_{0}$ inside $\mathfrak{m}$, and in fact all spaces $\mathfrak{m}_{i}$ are pairwise perpendicular with respect to the Killing form $B(X, Y):=-\operatorname{Re}(\operatorname{tr} X Y) / 2$. Hence, the following formula

$$
\mathfrak{g}_{s, y}:=\left.\frac{1}{s^{2}} B\right|_{\mathfrak{m}_{0}}+\left.B\right|_{\mathfrak{m}_{1}}+\left.\frac{1}{y} B\right|_{\mathfrak{m}_{2}}+\left.\frac{1}{y} B\right|_{\mathfrak{m}_{3}}
$$

defines a two-parameter family of metrics on $N(1,1):=\mathrm{SU}(3) / S^{1}$. It is a subfamily of the family considered in [2, p.109 ff]; in particular, $(s=1, y=2)$ corresponds to the 3 -Sasakian metric that has three Killing spinors with Killing number $1 / 2$, and $(s=$ $1, y=2 / 5)$ is the Einstein metric with one Killing spinor with Killing number $-3 / 10$ (see [2, Thm 12, p.116]). An orthonormal basis of $\mathfrak{m}$ is given by

$$
X_{1}=A_{12}, X_{2}=\tilde{A}_{12}, X_{3}=\sqrt{y} A_{13}, X_{4}=\sqrt{y} \tilde{A}_{13}, X_{5}=\sqrt{y} A_{23}, X_{6}=\sqrt{y} \tilde{A}_{23},
$$

and $X_{7}=s \cdot L / 3$. The isotropy representation $\operatorname{Ad}(\theta)$ leaves the vectors $X_{1}, X_{2}$ and $X_{7}$ invariant, and acts as a rotation by $3 \theta$ in the $\left(X_{3}, X_{4}\right)$-plane and in the $\left(X_{5}, X_{6}\right)$-plane. We use the standard realization of the 8-dimensional $\operatorname{Spin}(7)$-representation $\Delta_{7}$ as given in [2, p.97] or [13, p.13], and denote by $\psi_{i}, i=1, \ldots 8$ its basis $\left(u_{i}\right.$ in the notation of [2]). One then checks that $\psi_{3}, \psi_{4}, \psi_{5}$ and $\psi_{6}$ are fixed under the lift $\tilde{A d}(\theta)$ of the isotropy representation to $\operatorname{Spin}(7)$. Thus, they define constant sections in the spinor 
bundle $S=\mathrm{SU}(3) \times{ }_{\text {Ad }} \Delta_{7}$. The Levi-Civita connection of $N(1,1)$ is described by a map $\Lambda: \mathfrak{m} \mapsto \mathfrak{s o}(7)$, whose lift $\tilde{\Lambda}: \mathfrak{m} \mapsto \mathfrak{s p i n}(7)$ is given by $^{3}([2$, p.112])

$$
\begin{aligned}
& \tilde{\Lambda}\left(X_{1}\right)=+\frac{1}{2 s} e_{2} \cdot e_{7}-\left[\frac{1}{2}-\frac{y}{4}\right]\left[e_{3} \cdot e_{5}+e_{4} \cdot e_{6}\right] \\
& \tilde{\Lambda}\left(X_{2}\right)=-\frac{1}{2 s} e_{1} \cdot e_{7}-\left[\frac{1}{2}-\frac{y}{4}\right]\left[e_{4} \cdot e_{5}-e_{3} \cdot e_{6}\right] \\
& \tilde{\Lambda}\left(X_{3}\right)=+\frac{y}{4 s} e_{4} \cdot e_{7}-\frac{y}{4}\left[e_{2} \cdot e_{6}-e_{1} \cdot e_{5}\right] \\
& \tilde{\Lambda}\left(X_{4}\right)=-\frac{y}{4 s} e_{3} \cdot e_{7}+\frac{y}{4}\left[e_{1} \cdot e_{6}+e_{2} \cdot e_{5}\right] \\
& \tilde{\Lambda}\left(X_{5}\right)=-\frac{y}{4 s} e_{6} \cdot e_{7}-\frac{y}{4}\left[e_{1} \cdot e_{3}+e_{2} \cdot e_{4}\right] \\
& \tilde{\Lambda}\left(X_{6}\right)=+\frac{y}{4 s} e_{5} \cdot e_{7}-\frac{y}{4}\left[e_{1} \cdot e_{4}-e_{2} \cdot e_{3}\right] \\
& \tilde{\Lambda}\left(X_{7}\right)=\frac{s}{2}\left[2 e_{1} \cdot e_{2}+e_{3} \cdot e_{4}-e_{5} \cdot e_{6}\right]-\frac{1}{2 s} e_{1} \cdot e_{2}-\frac{y}{4 s} e_{3} \cdot e_{4}+\frac{y}{4 s} e_{5} \cdot e_{6} .
\end{aligned}
$$

We now make the following Ansatz for an algebraic 3-form on $\mathfrak{m}$,

$$
\begin{aligned}
\mathrm{T} & =\alpha X_{1} \wedge X_{3} \wedge X_{5}+\beta X_{1} \wedge X_{4} \wedge X_{6}+\gamma X_{2} \wedge X_{4} \wedge X_{5}+\delta X_{2} \wedge X_{3} \wedge X_{6} \\
& +\mu X_{1} \wedge X_{2} \wedge X_{7}+\nu X_{3} \wedge X_{4} \wedge X_{7}+\eta X_{5} \wedge X_{6} \wedge X_{7} .
\end{aligned}
$$

For notational convenience, we shall write $X_{i j k}$ for $X_{i} \wedge X_{j} \wedge X_{k}$, and similarly for forms of any degree. In order to define a global form on $N(1,1)$, an algebraic form on $\mathfrak{m}$ needs to be invariant under the isotropy representation. This is true for $X_{127}, X_{347}$, and $X_{567}$, whereas for example $X_{135}$ does not exist globally. However, one easily checks that the two 2-forms $X_{35}+X_{46}, X_{45}-X_{36}$ are isotropy invariant, and this will suffice to check that all forms to follow are indeed well-defined on $N(1,1)$. In any event, $\left.X_{1}\right\lrcorner \mathrm{T}$ acts on algebraic spinors by Clifford multiplication with $\alpha e_{3} \cdot e_{5}+\beta e_{4} \cdot e_{6}+\mu e_{2} \cdot e_{7}$, and similarly for $X_{2}, \ldots, X_{7}$.

Proposition 8.1. The spinor field $\psi_{3}$ satisfies the equation $\left.\nabla_{X}^{g} \psi_{3}+(X\lrcorner \mathrm{T}\right) \cdot \psi_{3}=0$ exactly for one 3 -form $\mathrm{T}:=\mathrm{T}_{3}$,

$$
\begin{aligned}
\mathrm{T}_{3} & :=\left[\frac{1}{2}-\frac{y}{4}+\frac{1+y}{6 s}-\frac{s}{3}\right]\left[X_{135}+X_{146}\right]+\left[\frac{1}{2}-\frac{y}{4}-\frac{1+y}{6 s}+\frac{s}{3}\right]\left[X_{245}-X_{236}\right] \\
& +\left[\frac{2 y-1}{6 s}-\frac{2 s}{3}\right] X_{127}+\left[\frac{4+y}{12 s}-\frac{2 s}{3}\right]\left[X_{347}-X_{567}\right] .
\end{aligned}
$$

Proof. A computer computation yields that the overdetermined system of equations $\left.\nabla_{X_{i}}^{g} \psi_{3}+\left(X_{i}\right\lrcorner \mathrm{T}\right) \cdot \psi_{3}=0$ (for $\left.i=1, \ldots, 7\right)$ reduces to a linear system of seven equations in the seven variables $\alpha, \ldots, \eta$ with two free parameters $s, y>0$ :

$$
\begin{gathered}
\frac{1}{2 s}+1-\frac{y}{2}-\alpha-\beta+\mu=0,-\frac{1}{2 s}+1-\frac{y}{2}-\gamma+\delta-\mu=0, \frac{y}{4 s}-\alpha-\delta+\nu=0, \frac{y}{4 s}-\beta+\gamma+\nu=0, \\
-\frac{y}{4 s}+\alpha-\gamma+\eta=0,-\frac{y}{4 s}+\beta+\delta+\eta=0,2 s-\frac{1+y}{2 s}+\mu+\nu-\eta=0 .
\end{gathered}
$$

One then verifies that the coefficients given in the proposition are its unique solution.

\footnotetext{
${ }^{3}$ Notice the following typo in the reference: the right definition of $d$ on page 112 is $d:=\sqrt{x y / z}+$ $\sqrt{y z / x}-\sqrt{x z / y}$. For our metrics, $x=1$ and $y=z$.
} 
Proposition 8.2. The spinor field $\psi_{4}$ satisfies the equation $\left.\nabla_{X}^{g} \psi_{4}+(X\lrcorner \mathrm{T}\right) \cdot \psi_{4}=0$ exactly for one 3 -form $\mathrm{T}:=\mathrm{T}_{4}$,

$$
\begin{aligned}
\mathrm{T}_{4} & :=\left[\frac{1}{2}-\frac{y}{4}-\frac{1+y}{6 s}+\frac{s}{3}\right]\left[X_{135}+X_{146}\right]+\left[\frac{1}{2}-\frac{y}{4}+\frac{1+y}{6 s}-\frac{s}{3}\right]\left[X_{245}-X_{236}\right] \\
& +\left[\frac{2 y-1}{6 s}-\frac{2 s}{3}\right] X_{127}+\left[\frac{4+y}{12 s}-\frac{2 s}{3}\right]\left[X_{347}-X_{567}\right] .
\end{aligned}
$$

Proof. The linear system determined by $\left.\nabla_{X_{i}}^{g} \psi_{4}+\left(X_{i}\right\lrcorner \mathrm{T}\right) \cdot \psi_{4}=0$ reads as

$$
\begin{gathered}
\frac{1}{2 s}-1+\frac{y}{2}+\alpha+\beta+\mu=0, \frac{1}{2 s}+1-\frac{y}{2}-\gamma+\delta+\mu=0, \frac{y}{4 s}+\alpha+\delta+\nu=0, \frac{y}{4 s}+\beta-\gamma+\nu=0, \\
\frac{y}{4 s}+\alpha-\gamma-\eta=0, \frac{y}{4 s}+\beta+\delta-\eta=0,-2 s+\frac{1+y}{2 s}-\mu-\nu+\eta=0 .
\end{gathered}
$$

Its unique solution leads to the formulas above.

Proposition 8.3. The spinor field $\psi_{5}$ satisfies the equation $\left.\nabla_{X}^{g} \psi_{5}+(X\lrcorner \mathrm{T}\right) \cdot \psi_{5}=0$ exactly for one 3 -form $\mathrm{T}:=\mathrm{T}_{5}$,

$$
\begin{aligned}
\mathrm{T}_{5} & :=\left[\frac{1}{6}+\frac{y}{12}+\frac{y-1}{6 s}\right]\left[X_{135}+X_{146}+X_{245}-X_{236}\right]+\left[\frac{2}{3}-\frac{2 y}{3}-\frac{2 y+1}{6 s}\right] X_{127} \\
& +\left[\frac{1}{3}-\frac{y}{3}-\frac{4-y}{12 s}\right]\left[X_{347}-X_{567}\right] .
\end{aligned}
$$

Proof. The linear system $\left.\nabla_{X_{i}}^{g} \psi_{5}+\left(X_{i}\right\lrcorner \mathrm{T}\right) \cdot \psi_{5}=0$ is of slightly different type,

$$
\begin{gathered}
\frac{y}{4 s}+\frac{y}{2}-\alpha+\delta+\nu=0, \frac{y}{4 s}+\frac{y}{2}-\beta-\gamma+\nu=0, \frac{y}{4 s}+\frac{y}{2}-\alpha-\gamma-\eta=0, \frac{y}{4 s}+\frac{y}{2}-\beta+\delta-\eta=0, \\
\frac{1}{2 s}-1+\frac{y}{2}+\gamma-\delta+\mu=0, \frac{1}{2 s}-1+\frac{y}{2}+\alpha+\beta+\mu=0, \frac{y-1}{2 s}+\mu-\nu+\eta=0 .
\end{gathered}
$$

The main reason for this is that $\psi_{5}$ and $\psi_{6}$ span the kernel of the first summand of $\tilde{\Lambda}\left(X_{7}\right)$, hence the last equation contains no term linear in $s$.

Proposition 8.4. The spinor field $\psi_{6}$ satisfies the equation $\left.\nabla_{X}^{g} \psi_{6}+(X\lrcorner \mathrm{T}\right) \cdot \psi_{6}=0$ exactly for one 3 -form $\mathrm{T}:=\mathrm{T}_{6}$,

$$
\begin{aligned}
\mathrm{T}_{6} & :=\left[\frac{1}{6}+\frac{y}{12}-\frac{y-1}{6 s}\right]\left[X_{135}+X_{146}+X_{245}-X_{236}\right]+\left[-\frac{2}{3}+\frac{2 y}{3}-\frac{2 y+1}{6 s}\right] X_{127} \\
& +\left[-\frac{1}{3}+\frac{y}{3}-\frac{4-y}{12 s}\right]\left[X_{347}-X_{567}\right] .
\end{aligned}
$$

Proof. The linear system $\left.\nabla_{X_{i}}^{g} \psi_{6}+\left(X_{i}\right\lrcorner \mathrm{T}\right) \cdot \psi_{6}=0$ is

$$
\begin{aligned}
& \frac{y}{4 s}-\frac{y}{2}+\alpha-\delta+\nu=0, \frac{y}{4 s}-\frac{y}{2}+\beta+\gamma+\nu=0, \frac{y}{4 s}-\frac{y}{2}+\alpha+\gamma-\eta=0, \frac{y}{4 s}-\frac{y}{2}+\beta-\delta-\eta=0, \\
& -\frac{1}{2 s}-1+\frac{y}{2}+\gamma-\delta-\mu=0,-\frac{1}{2 s}-1+\frac{y}{2}+\alpha+\beta-\mu=0, \frac{1-y}{2 s}-\mu+\nu-\eta=0 .
\end{aligned}
$$

Remark 8.1. For $s=y=1$, all four 3 -forms $\mathrm{T}_{3}, \ldots, \mathrm{T}_{6}$ coincide, reflecting the fact that the undeformed metric has $\psi_{3}, \ldots, \psi_{6}$ as parallel spinors for the connection defined by

$$
\mathrm{T}:=\frac{1}{4}\left[X_{135}+X_{146}+X_{245}-X_{236}\right]-\frac{1}{2} X_{127}-\frac{1}{4}\left[X_{347}-X_{567}\right] .
$$


The 3 -forms $\mathrm{T}_{3}$ and $\mathrm{T}_{4}$ are equal for the family of metrics defined by $2 s^{2}=1+y$, whereas $\mathrm{T}_{5}=\mathrm{T}_{6}$ as soon as $y=1$. Even more interestingly, there exists a metric for which $\mathrm{T}_{3}=-\mathrm{T}_{4}$ :

Proposition 8.5. Consider the metric $g_{s_{0}, y_{0}}$ on $N(1,1)$ defined by $s_{0}=\sqrt{3} / 2$ and $y_{0}=2$, and the 3 -form

$$
\mathrm{T}:=\sqrt{3} / 6\left(X_{135}+X_{146}-X_{245}+X_{236}\right) .
$$

Then, $\psi_{3}$ is parallel with respect to the connection $\nabla^{4 \cdot \mathrm{T}}$, and $\psi_{4}$ is parallel with respect to the connection $\nabla^{-4 \cdot \mathrm{T}}$. Furthermore, both connections are not flat.

It is a subtle and computationally difficult question in as much $\mathrm{T}$ can be adapted to a given spinor in order to make it parallel. For this, a more systematic approach is required. There are precisely 13 isotropy invariant 3 -forms on $\mathfrak{m}$, hence the most general 3 -form we can consider is a linear combination of

$$
\begin{gathered}
X_{135}+X_{146}, X_{235}+X_{246}, X_{357}+X_{467}, X_{145}-X_{136}, X_{245}-X_{236}, X_{457}-X_{367}, \\
X_{127}, X_{347}, X_{567}, X_{134}, X_{234}, X_{156}, X_{256} .
\end{gathered}
$$

We studied the question whether there exists a continuous family of 3 -forms $\mathrm{T}_{a, b}$ of this general type such that a given linear combination $a \psi_{3}+b \psi_{5}$ is parallel with respect to $\nabla^{\mathrm{T}_{a, b}}$. It turns out that this is possible if and only if $s=y$. We state the result of this lengthy calculation without proof.

Proposition 8.6. The spinor field $\psi_{a, b}:=a \cdot \psi_{3}+b \cdot \psi_{5}, a b \neq 0$, satisfies the equation $\left.\nabla_{X}^{g} \psi_{a, b}+(X\lrcorner \mathrm{T}_{a, b}\right) \cdot \psi_{a, b}=0$ if and only if $s=y$ and if $\mathrm{T}_{a, b}$ is given by

$$
\begin{aligned}
\mathrm{T}_{a, b} & =\frac{a^{2}\left(-7 s^{2}+8 s+2\right)+b^{2}\left(s^{2}+4 s-2\right)}{12 s\left(a^{2}+b^{2}\right)}\left[X_{135}+X_{146}\right]+\frac{s^{2}+4 s-2}{12 s}\left[X_{245}-X_{236}\right] \\
& +\frac{a^{2}\left(-8 s^{2}+s+4\right)+b^{2}\left(-4 s^{2}+5 s-4\right)}{12 s\left(a^{2}+b^{2}\right)}\left[X_{347}-X_{567}\right]+\frac{-4 s^{2}+2 s-1}{6 s} X_{127} \\
& +\frac{a b\left(-2 s^{2}+s+1\right)}{3 s\left(a^{2}+b^{2}\right)}\left[X_{134}-X_{156}\right]+\frac{a b\left(s^{2}+s-2\right)}{3 s\left(a^{2}+b^{2}\right)}\left[X_{357}+X_{467}\right]
\end{aligned}
$$

For $a b=0$, this differential form $\mathrm{T}_{a, b}$ is again a linear combination of the seven basic 3 -forms we started with, and coincides indeed for $a=1, b=0$ and $a=0, b=1$ with the 3 -forms $\mathrm{T}_{3}, \mathrm{~T}_{5}$ evaluated at the parameter value $s=y$, respectively. Remark that the connections with torsion $\mathrm{T}_{a, b}$ constitute a $S^{1}$-parameter family of connections admitting parallel spinors on the same Riemannian manifold. The 3-Sasakian metric $(s=1, y=2)$ and the Einstein metric $(s=1, y=2 / 5)$ are of particular interest. For theoretical reasons to be explained in the next section, both must admit a family of torsion forms such that the three Killing spinors of the 3-Sasakian metric $\left(\psi_{3}, \psi_{4}, \psi_{6}\right.$ in our notation) are parallel with respect to the connection it defines. In fact, such a family exists for $s=1$ and arbitrary $y$ (but not for arbitrary $s$ ).

Proposition 8.7. For the metrics $g_{s, y}$ on $N(1,1)$, the spinor field $\psi_{a, b, c}:=a \psi_{3}+b \psi_{4}+$ $c \psi_{6}, a b c \neq 0$, satisfies the equation $\left.\nabla_{X}^{g} \psi_{a, b, c}+(X\lrcorner \mathrm{T}_{a, b, c}\right) \cdot \psi_{a, b, c}=0$ if and only if $s=1$ and if $\mathrm{T}_{a, b, c}$ is given by

$$
\begin{aligned}
\mathrm{T}_{a, b, c} & =P(a, b, c)\left[X_{567}-X_{347}\right]+P(a, c, b)\left[X_{135}+X_{146}\right]+P(b, c, a)\left[X_{245}-X_{236}\right] \\
& +Q(a, b, c)\left[X_{235}+X_{246}+X_{145}-X_{136}\right]+Q(b, c, a)\left[X_{357}+X_{467}+X_{156}-X_{134}\right] \\
& +Q(a, c, b)\left[X_{457}-X_{367}+X_{234}-X_{256}\right]+\frac{2 y-5}{6} X_{127}
\end{aligned}
$$


with the following definitions for the coefficients $P$ and $Q$ :

$$
P(a, b, c):=\frac{\left(a^{2}+b^{2}\right)(4-y)+c^{2}(8-5 y)}{12\left(a^{2}+b^{2}+c^{2}\right)}, \quad Q(a, b, c):=\frac{a b(y-1)}{3\left(a^{2}+b^{2}+c^{2}\right)} .
$$

Let us discuss the spinor fields $\psi_{3}$ and $\psi_{5}$ from the point of view of $\mathrm{G}_{2}$-geometry. In general, a spinor field $\psi$ of length one defines on a 7-dimensional Riemannian manifold a 3-form of general type by the formula (see [2], [19])

$$
\omega(X, Y, Z):=-\langle X \cdot Y \cdot Z \cdot \psi, \psi\rangle .
$$

Computing the forms of the spinors $\psi_{3}, \psi_{5}$ we obtain

$$
\begin{aligned}
& \omega_{3}=-X_{127}+X_{135}+X_{146}+X_{236}-X_{245}-X_{347}+X_{567}, \\
& \omega_{5}=-X_{127}-X_{135}-X_{146}+X_{236}-X_{245}+X_{347}-X_{567} .
\end{aligned}
$$

The connections $\nabla^{3}$ and $\nabla^{5}$ with torsion forms $4 \cdot \mathrm{T}_{3}$ and $4 \cdot \mathrm{T}_{5}$ preserve the $\mathrm{G}_{2}$-structures $\omega_{3}$ and $\omega_{5}$, respectively. Moreover, a direct computation yields the formulas

$$
\begin{aligned}
\left(\mathrm{T}_{3}, \omega_{3}\right) & =\frac{4 \cdot s^{2}+y+1}{6 \cdot s}>0, \\
\left(\mathrm{~T}_{5}, \omega_{5}\right) & =-\frac{4 \cdot s+2 \cdot s \cdot y+y-1}{6 \cdot s} .
\end{aligned}
$$

Since the connection preserving a $\mathrm{G}_{2}$-structure is unique (see [15]), the $\mathrm{G}_{2}$-structures $\omega_{3}$ and $\omega_{5}$ are not equivalent. We remark that $\omega_{3}$ and $\omega_{5}$ are cocalibrated $\mathrm{G}_{2}$-structures,

$$
d * \omega_{3}=0, \quad d * \mathrm{~T}_{3}=0, \quad d * \omega_{5}=0, \quad d * \mathrm{~T}_{5}=0 .
$$

Indeed, for any vector, the inner product $X\lrcorner * \omega_{3}$ is orthogonal to $7 \cdot \mathrm{T}_{3}-\left(\mathrm{T}_{3}, \omega_{3}\right) \cdot \omega_{3}$. The formula expressing the torsion form $\mathrm{T}$ of an admissible $\mathrm{G}_{2}$-structure by the 3 -form $\omega$ ( see [15] and [17]) yields now $d * \omega_{3}=0$ immediately. The codifferential of the torsion form is given by the formula (see [15] and [17])

$$
4 \cdot d * \mathrm{~T}_{3}=d \lambda_{3} \wedge * \omega_{3}, \quad \lambda_{3}=\left(4 \cdot \mathrm{T}_{3}, \omega_{3}\right) .
$$

In our example the function $\lambda_{3}$ is constant, i.e., $d * \mathrm{~T}_{3}=0$. The same argument applies for $\omega_{5}$. The class of all cocalibrated $\mathrm{G}_{2}$-structures splits into the sum $\mathcal{W}_{1} \oplus \mathcal{W}_{3}$ of a 1 dimensional class $\mathcal{W}_{1}$ (the so called nearly parallel $\mathrm{G}_{2}$-structures) and a 27 -dimensional class $\mathcal{W}_{3}$ (see [1]). Nearly parallel $\mathrm{G}_{2}$-structures are characterized by the condition that the torsion form $\mathrm{T}$ of its unique connection is proportional to $\omega$. On the other side, the $\mathrm{G}_{2}$-structures of type $\mathcal{W}_{3}$ are the cocalibrated structures such that $\mathrm{T}$ and $\omega$ are orthogonal, $(\mathrm{T}, \omega)=0$ (see [15]). Using this characterization we obtain immediately

Proposition 8.8. The $\mathrm{G}_{2}$-structure $\omega_{3}$ is nearly parallel if and only if $s=1$ and $y=2$. The $\mathrm{G}_{2}$-structure $\omega_{3}$ is never of type $\mathcal{W}_{3}$. The $\mathrm{G}_{2}$-structure $\omega_{5}$ is nearly parallel if and only if $s=1$ and $y=2 / 5$. This metric is a universal deformation of the 3-Sasakian metric (see [19]). The $\mathrm{G}_{2}$-structure $\omega_{5}$ is of type $\mathcal{W}_{3}$ if and only if $2 \cdot s \cdot(2+y)=1-y$. In general, the scalar curvatures $\mathrm{Scal}^{g}, \mathrm{Scal}^{\nabla}$ of a cocalibrated $\mathrm{G}_{2}$-structure $\left(M^{7}, g, \omega\right)$ can be expressed by its torsion form $\mathrm{T}$ (see [17]) :

$\mathrm{Scal}^{g}=2 \cdot(\mathrm{T}, \omega)^{2}-\frac{1}{2} \cdot\|\mathrm{T}\|^{2}, \quad \mathrm{Scal}^{\nabla}=\mathrm{Scal}^{g}-\frac{3}{2} \cdot\|\mathrm{T}\|^{2}=2 \cdot(\mathrm{T}, \omega)^{2}-2 \cdot\|\mathrm{T}\|^{2}$.

We use the forms $\omega_{3}, 4 \cdot \mathrm{T}_{3}$ as well as the forms $\omega_{5}, 4 \cdot \mathrm{T}_{5}$ in order to compute the Riemannian scalar curvature of the metric depending on the parameters $s, y$. In both 
cases the result is the same :

$$
\mathrm{Scal}^{g}=8+24 y-2 y^{2}-\frac{2+y^{2}}{s^{2}} .
$$

In a similar way we compute the scalar curvature of the connection $\nabla^{3}$ and $\nabla^{5}$ :

$$
\begin{gathered}
\mathrm{Scal}^{3}=-\frac{4}{3 s^{2}} \cdot\left(8+32 s^{4}+4 y+5 y^{2}+2 s^{2}\left(-4-28 y+3 y^{2}\right)\right) \\
\mathrm{Scal}^{5}=-\frac{4}{3 s^{2}} \cdot\left(8-4 y+5 y^{2}+8 s\left(-2+y+y^{2}\right)+2 s^{2}\left(4-20 y+7 y^{2}\right)\right) .
\end{gathered}
$$

In particular, we obtain a family of cocalibrated $\mathrm{G}_{2}$-structures on $N(1,1)$ with vanishing scalar curvature of the associated connection. Moreover, a numerical computation yields that there exist two pairs of parameters where both scalar curvatures Scal ${ }^{3}$ and $\mathrm{Scal}^{5}$ vanish, namely $(s, y) \approx(0.62066,0.852508)$ and $(1.49934,1.66564)$. The Ricci tensor Ric $^{\nabla}$ of the canonical connection of a $\mathrm{G}_{2}$-structure $\left(M^{7}, g, \omega\right)$ can be expressed by the derivative $d \mathrm{~T}$ of the torsion form (see [15]),

$$
\left.\left.\operatorname{Ric}^{\nabla}\left(X_{i}, X_{j}\right)=\frac{1}{2} \cdot\left(X_{i}\right\lrcorner d \mathrm{~T}, X_{j}\right\lrcorner * \omega\right) .
$$

Using the commutator relations in the Lie algebra we compute the exterior derivatives

$$
\begin{aligned}
& d X_{1}=-2 s \cdot X_{27}+y \cdot\left(X_{35}+X_{46}\right), \\
& d X_{2}=2 s \cdot X_{17}+y \cdot\left(X_{45}-X_{36}\right), \\
& d X_{7}=-\frac{2}{s} \cdot X_{12}-\frac{y}{s} \cdot\left(X_{34}-X_{56}\right) .
\end{aligned}
$$

The torsion form $\mathrm{T}_{3}$ can be written as

$$
\begin{aligned}
\mathrm{T}_{3} & =-\frac{(y-2)\left(5 s^{2}-1-y\right)}{3 s y} X_{127}+\frac{1}{y}\left[\frac{1}{2}-\frac{y}{4}+\frac{1+y}{6 s}-\frac{s}{3}\right] X_{1} \wedge d X_{1} \\
& +\frac{1}{y}\left[\frac{1}{2}-\frac{y}{4}-\frac{1+y}{6 s}+\frac{s}{3}\right] X_{2} \wedge d X_{2}-\frac{s}{y}\left[\frac{4+y}{12 s}-\frac{2 s}{3}\right] X_{7} \wedge d X_{7} .
\end{aligned}
$$

We can now compute the exterior derivative as well as the Ricci tensor. Let us discuss the cases $(s, y)=(1,4)$ and $(s, y)=(\sqrt{3 / 2}, 2)$ where the formulas simplify.

Example 8.1. In case of $s=1$ and $y=4$ we obtain

$$
\mathrm{T}_{3}=-\frac{1}{4} X_{2} \wedge d X_{2}, \quad d \mathrm{~T}_{3}=8 X_{3456}-4 X_{1457}+4 X_{1367} .
$$

The scalar curvatures are $\mathrm{Scal}^{3}=0$ and $\mathrm{Scal}^{g}=54$. Moreover, we obtain

$$
-2 \cdot \sigma_{\mathrm{T}_{3}}=\left(\mathrm{T}_{3}^{2}\right)_{4}=\frac{1}{4} \cdot d \mathrm{~T}_{3},
$$

i.e., $d \mathrm{~T}_{3}$ is proportional to $\sigma_{\mathrm{T}_{3}}$ (see Theorem 7.1).

Example 8.2. In case of $s=\sqrt{3 / 2}$ and $y=2$ we obtain

$$
\mathrm{T}_{3}=\frac{1}{4} X_{7} \wedge d X_{7}, \quad d \mathrm{~T}_{3}=\frac{4}{3}\left(X_{1234}-X_{1256}-X_{3456}\right) .
$$

The scalar curvatures $\mathrm{Scal}^{3}$ and $\mathrm{Scal}^{g}$ are positive. 
The 3 -form $\omega_{a, b}$ corresponding to the spinor $a \cdot \psi_{3}+b \cdot \psi_{5}$ is given by the formula

$$
\begin{aligned}
\omega_{a, b}= & -\left(a^{2}+b^{2}\right)\left(X_{127}-X_{236}+X_{245}\right)+2 a b\left(X_{134}-X_{156}+X_{357}+X_{467}\right) \\
+ & \left(a^{2}-b^{2}\right)\left(X_{135}+X_{146}-X_{347}+X_{567}\right) .
\end{aligned}
$$

We compute the inner product with the torsion form of Proposition 8.6 :

$$
\left(\mathrm{T}_{a, b}, \omega_{a, b}\right)=\frac{b^{2}\left(1-5 s-2 s^{2}\right)+a^{2}\left(1+s+4 s^{2}\right)}{6 s} .
$$

In particular, the $\mathrm{G}_{2}$-structure is of pure type $\mathcal{W}_{3}$ if and only if

$$
b^{2}\left(1-5 s-2 s^{2}\right)+a^{2}\left(1+s+4 s^{2}\right)=0, \quad y=s .
$$

Finally, we will construct non trivial 4-forms on $N(1,1)$ such that the underlying connections admit parallel spinors. Remark that spinorial connections related to 4 -forms are of completely different type. For example, they do not preserve the hermitian product of spinors and, in general, the holonomy group of a spinorial connection of that type is non compact. Nevertheless, for the family of metrics $g_{s, y}$, the qualitative results are quite similar to those for 3-forms, though they cannot be deduced one from each other.

Theorem 8.1. On $N(1,1)$ with the metric $g_{s, y}$, there exist four spinorial connections defined by 4-forms admitting $\psi_{3}, \psi_{4}, \psi_{5}$ and $\psi_{6}$ as parallel spinors, respectively; and there exists, for $s=1$, arbitrary $y$ and any linear combination of $\psi_{3}, \psi_{4}, \psi_{6}$ precisely one 4 form which makes this particular combination parallel.

Again, the exposition of results will make the statement more precise. No proofs will be given, since they are similar to the corresponding computations for 3 -forms. With the same notations as before, consider now the following Ansatz for a global 4-form :

$$
\begin{aligned}
R & =\alpha X_{1234}+\beta X_{1256}+\gamma X_{3456}+\delta X_{1347}+\varepsilon X_{1567}+\xi X_{2347}+\mu X_{2567} \\
& +\nu\left(X_{1235}+X_{1246}\right)+\lambda\left(X_{1357}+X_{1467}\right)+\eta\left(X_{1245}-X_{1236}\right) \\
& +\omega\left(X_{1457}-X_{1367}\right)+\pi\left(X_{2457}-X_{2367}\right)+\varrho\left(X_{2357}+X_{2467}\right) .
\end{aligned}
$$

Proposition 8.9. The spinor field $\psi_{3}$ satisfies the equation $\left.\nabla_{X}^{g} \psi_{3}+(X\lrcorner R\right) \cdot \psi_{3}=0$ if and only if $R=R_{3}$, with

$$
\begin{aligned}
R_{3} & :=\left[\frac{y}{4}-\frac{1}{2}+\frac{1+y}{8 s}-\frac{s}{2}\right]\left[X_{1457}-X_{1367}\right]+\left[\frac{1}{2}-\frac{y}{4}+\frac{1+y}{8 s}-\frac{s}{2}\right]\left[X_{2357}+X_{2467}\right] \\
& +\left[-\frac{s}{2}+\frac{y+3}{8 s}\right]\left[X_{1234}-X_{1256}\right]+\left[\frac{s}{2}+\frac{1-3 y}{8 s}\right] X_{3456} .
\end{aligned}
$$

Proposition 8.10. The spinor field $\psi_{4}$ satisfies the equation $\left.\nabla_{X}^{g} \psi_{4}+(X\lrcorner R\right) \cdot \psi_{4}=0$ if and only if $R=R_{4}$, with

$$
\begin{aligned}
R_{4} & :=\left[\frac{y}{4}-\frac{1}{2}-\frac{1+y}{8 s}+\frac{s}{2}\right]\left[X_{1457}-X_{1367}\right]+\left[\frac{1}{2}-\frac{y}{4}-\frac{1+y}{8 s}+\frac{s}{2}\right]\left[X_{2357}+X_{2467}\right] \\
& +\left[-\frac{s}{2}+\frac{y+3}{8 s}\right]\left[X_{1234}-X_{1256}\right]+\left[\frac{s}{2}+\frac{1-3 y}{8 s}\right] X_{3456} .
\end{aligned}
$$

Proposition 8.11. The spinor field $\psi_{5}$ satisfies the equation $\left.\nabla_{X}^{g} \psi_{5}+(X\lrcorner R\right) \cdot \psi_{5}=0$ if and only if $R=R_{5}$, with

$$
\begin{aligned}
R_{5} & :=\left[\frac{1}{2}-\frac{y}{4}+\frac{y-3}{8 s}\right]\left[X_{1234}-X_{1256}\right]+\left[-\frac{1}{2}+\frac{3 y}{4}+\frac{1+3 y}{8 s}\right] X_{3456} \\
& +\frac{1-y}{8 s}\left[\left(X_{1457}-X_{1367}\right)-\left(X_{2357}+X_{2467}\right)\right] .
\end{aligned}
$$


Proposition 8.12. The spinor field $\psi_{6}$ satisfies the equation $\left.\nabla_{X}^{g} \psi_{6}+(X\lrcorner R\right) \cdot \psi_{6}=0$ if and only if $R=R_{6}$, with

$$
\begin{aligned}
R_{6} & :=\left[-\frac{1}{2}+\frac{y}{4}+\frac{y-3}{8 s}\right]\left[X_{1234}-X_{1256}\right]+\left[\frac{1}{2}-\frac{3 y}{4}+\frac{1+3 y}{8 s}\right] X_{3456} \\
& +\frac{y-1}{8 s}\left[\left(X_{1457}-X_{1367}\right)-\left(X_{2357}+X_{2467}\right)\right] .
\end{aligned}
$$

Remark 8.2. For $s=y=1$, no two of the four 4 -forms $R_{3}, \ldots, R_{6}$ coincide, reflecting the different behavior of spinorial connections defined by 4 -forms when compared to connections defined by 3 -forms. The 4 -forms $R_{3}$ and $R_{4}$ are equal for the family of metrics defined by $4 s^{2}=1+y$, whereas $R_{5}$ and $R_{6}$ are never equal. As for 3 -forms, there exists a metric for which $R_{3}=-R_{4}$ :

Proposition 8.13. Consider the metric $g_{s_{0}, y_{0}}$ on $N(1,1)$ defined by $s_{0}=\sqrt{5} / 2$ and $y_{0}=2$, and the 4 -form

$$
R:=-\sqrt{5} / 10\left[\left(X_{1457}-X_{1367}\right)+\left(X_{2357}+X_{2467}\right)\right] .
$$

Then, $\psi_{3}$ is parallel with respect to the connection $\nabla^{R}$, and $\psi_{4}$ is parallel with respect to the connection $\nabla^{-R}$. Furthermore, both connections are not flat.

Proposition 8.14. For the metrics $g_{s, y}$ on $N(1,1)$, the spinor field $\psi_{a, b, c}:=a \psi_{3}+$ $b \psi_{4}+c \psi_{6}, a b c \neq 0$, satisfies the equation $\left.\nabla_{X}^{g} \psi_{a, b, c}+(X\lrcorner R_{a, b, c}\right) \cdot \psi_{a, b, c}=0$ if and only if $s=1$ and if $R_{a, b, c}$ is given by

$$
\begin{aligned}
R_{a, b, c} & =P(a, b, c)\left[X_{1234}-X_{1256}\right]-P(a, c, b)\left[X_{2467}+X_{2357}\right]+P(b, c, a)\left[X_{1457}-X_{1367}\right] \\
& +Q(a, b, c)\left[\left(X_{2457}-X_{2367}\right)-\left(X_{1357}+X_{1467}\right)\right]+Q(b, c, a)\left[\left(X_{1235}+X_{1246}\right)+\right. \\
& \left.+\left(X_{2567}-X_{2347}\right)\right]+Q(a, c, b)\left[\left(X_{1567}-X_{1347}\right)-\left(X_{1245}-X_{1236}\right)\right]+\frac{5-3 y}{8} X_{3456},
\end{aligned}
$$

with the following definitions for the coefficients $P$ and $Q$ :

$$
P(a, b, c):=\frac{\left(a^{2}+b^{2}\right)(y-1)+c^{2}(3 y-7)}{8\left(a^{2}+b^{2}+c^{2}\right)}, \quad Q(a, b, c):=\frac{a b(y-3)}{4\left(a^{2}+b^{2}+c^{2}\right)} .
$$

\section{Torsion forms with parallel spinors on 3-Sasakian manifolds}

The Aloff-Wallach space $N(1,1)$ admits a 3-Sasakian structure, and some special torsion forms with parallel spinors discussed in Section 8 are closely related to the underlying contact structures of $N(1,1)$. This observation yielded the idea that any 3-Sasakian manifold should admit natural connections with skew-symmetric torsion and parallel spinors. In this section, we will make this remark precise. In particular, for a fixed 3-Sasakian metric, we will construct a whole family of connections with parallel spinors. The structure group of a 3-Sasakian geometry is the subgroup $\mathrm{SU}(2) \subset \mathrm{G}_{2} \subset \mathrm{SO}(7)$, the isotropy group of four spinors in dimension seven. In order to keep the realization of the spin representation we used in Section 8, we describe the subgroup SU(2) in such a way that the vectors $e_{1}, e_{2}, e_{7} \in \mathbb{R}^{7}$ are fixed. More precisely, the Lie algebra $\mathfrak{s u}(2)$ is generated by the following 2 -forms in $\mathbb{R}^{7}$ :

$$
e_{34}+e_{56}, \quad e_{35}-e_{46}, \quad e_{36}+e_{56} .
$$

The real spin representation $\Delta_{7}$ splits under the action of $\mathrm{SU}(2)$ into a 4-dimensional trivial representation $\Delta_{7}^{0}$ and the unique non trivial 4-dimensional representation $\Delta_{7}^{1}$. In 
our spin basis, the space $\Delta_{7}^{0}$ is spanned by the spinors $\psi_{3}, \psi_{4}, \psi_{5}, \psi_{6}$. We consider the following $\mathrm{SU}(2)$-invariant 2-forms on $\mathbb{R}^{7}$ :

$$
d e_{1}:=e_{35}+e_{46}, \quad d e_{2}:=e_{45}-e_{36}, \quad d e_{7}:=e_{34}-e_{56} .
$$

Using this notation, we introduce a family of invariant 3 -forms in $\mathbb{R}^{7}$ depending on 10 parameters,

$$
\mathrm{T}=\sum_{i, j=1,2,7} x_{i j} \cdot e_{i} \wedge d e_{j}+w \cdot e_{1} \wedge e_{2} \wedge e_{7}
$$

The key point of our considerations in this section is the following algebraic observation

Proposition 9.1. For any spinor $\psi \in \Delta_{7}^{0}$, there exists a unique invariant 3 -form $\mathrm{T}$ such that $\{X-2 \cdot X\lrcorner \mathrm{T}\} \cdot \psi=0$ holds for any vector $X \in \mathbb{R}^{7}$.

Proof. Given a spinor $\psi=a \psi_{3}+b \psi_{4}+c \psi_{5}+d \psi_{6}$, we solve the overdetermined system $(X-2 \cdot X\lrcorner \mathrm{T}) \cdot \psi=0$ with respect to the coefficients of the 3 -form. It turns out that a solution exists and is given by the following explicit formulas

$$
\begin{aligned}
& x_{11}=\frac{a^{2}-b^{2}-c^{2}+d^{2}}{6\left(a^{2}+b^{2}+c^{2}+d^{2}\right)}, x_{12}=\frac{a b+c d}{3\left(a^{2}+b^{2}+c^{2}+d^{2}\right)}, x_{17}=\frac{a c-b d}{3\left(a^{2}+b^{2}+c^{2}+d^{2}\right)}, \\
& x_{21}=\frac{a b-c d}{3\left(a^{2}+b^{2}+c^{2}+d^{2}\right)}, x_{22}=\frac{-a^{2}+b^{2}-c^{2}+d^{2}}{6\left(a^{2}+b^{2}+c^{2}+d^{2}\right)}, x_{27}=\frac{b c+a d}{3\left(a^{2}+b^{2}+c^{2}+d^{2}\right)}, \\
& x_{71}=\frac{a c+b d}{3\left(a^{2}+b^{2}+c^{2}+d^{2}\right)}, x_{72}=\frac{b c-a d}{3\left(a^{2}+b^{2}+c^{2}+d^{2}\right)}, x_{77}=\frac{-a^{2}-b^{2}+c^{2}+d^{2}}{6\left(a^{2}+b^{2}+c^{2}+d^{2}\right)} .
\end{aligned}
$$

and $w=-1 / 6$. The map $(a, b, c, d) \rightarrow x_{i j}(a, b, c, d)$ is the Veronese map from $\mathbb{P}^{3}$ into the sphere $S^{8}$ of radius $1 / 12$.

Consider a simply connected 3-Sasakian manifold $M^{7}$ of dimension seven and denote its three contact structures by $\eta_{1}, \eta_{2}$, and $\eta_{7}$. It is known that $M^{7}$ is then an Einstein space, and examples (also non homogeneous ones) can be found in the paper [4] by Boyer and Galicki. The tangent bundle of $M^{7}$ splits into the 3 -dimensional part spanned by $\eta_{1}, \eta_{2}, \eta_{7}$ and its 4-dimensional orthogonal complement. We restrict the exterior derivatives $d \eta_{1}, d \eta_{2}$ and $d \eta_{7}$ to this complement. In an adapted orthonormal frame, these forms coincide with the algebraic forms $d e_{1}, d e_{2}$ and $d e_{7}$. Now we apply Proposition 9.1] The space of Riemannian Killing spinors

$$
\nabla_{X}^{g} \psi+\frac{1}{2} \cdot X \cdot \psi=0
$$

is non trivial and has at least dimension three (see [18]). Moreover, the proof of this fact shows that all the Riemannian Killing spinors are sections in the subbundle corresponding to the $\mathrm{SU}(2)$-representation $\Delta_{7}^{0}$. Consequently, for any Killing spinor, there exists a unique torsion form $\mathrm{T}$ of the described type such that

$$
\left.\nabla_{X}^{\mathrm{T}} \psi=\nabla_{X}^{g} \psi+(X\lrcorner \mathrm{T}\right) \cdot \psi=0 .
$$

Theorem 9.1. Any 3 -Sasakian manifold in dimension seven admits a $\mathbb{P}^{2}$-parameter family of metric connections with skew-symmetric torsion and parallel spinors. The holonomy group of these connections is a subgroup of $\mathrm{G}_{2}$.

The space of $\mathrm{SU}(2)$-invariant 4 -forms on $\mathbb{R}^{7}$ has dimension ten,

$$
\mathrm{T}=\sum_{i, j, k=1,2,7} x_{i j k} \cdot e_{i} \wedge e_{j} \wedge d e_{k}+w \cdot e_{3} \wedge e_{4} \wedge e_{5} \wedge e_{6}
$$




\begin{tabular}{|l|l|l|l|l|}
\hline$\omega_{1,9}=\omega_{8,16}$ & $\omega_{1,10}=-\omega_{8,15}$ & $\omega_{1,11}=\omega_{8,14}$ & $\omega_{1,12}=\omega_{8,13}$ & $\omega_{1,13}=-\omega_{8,12}$ \\
\hline$\omega_{1,14}=-\omega_{8,11}$ & $\omega_{1,15}=\omega_{8,10}$ & $\omega_{1,16}=-\omega_{8,9}$ & $\omega_{2,9}=\omega_{8,15}$ & $\omega_{2,10}=\omega_{8,16}$ \\
\hline$\omega_{2,11}=-\omega_{8,13}$ & $\omega_{2,12}=\omega_{8,14}$ & $\omega_{2,13}=\omega_{8,11}$ & $\omega_{2,14}=-\omega_{8,12}$ & $\omega_{2,15}=-\omega_{8,9}$ \\
\hline$\omega_{2,16}=-\omega_{8,10}$ & $\omega_{3,9}=-\omega_{8,14}$ & $\omega_{3,10}=\omega_{8,13}$ & $\omega_{3,11}=\omega_{8,16}$ & $\omega_{3,12}=\omega_{8,15}$ \\
\hline$\omega_{3,13}=-\omega_{8,10}$ & $\omega_{3,14}=\omega_{8,9}$ & $\omega_{3,15}=-\omega_{8,12}$ & $\omega_{3,16}=-\omega_{8,11}$ & $\omega_{4,9}=-\omega_{8,13}$ \\
\hline$\omega_{4,10}=-\omega_{8,14}$ & $\omega_{4,11}=-\omega_{8,15}$ & $\omega_{4,12}=\omega_{8,16}$ & $\omega_{4,13}=\omega_{8,9}$ & $\omega_{4,14}=\omega_{8,10}$ \\
\hline$\omega_{4,15}=\omega_{8,11}$ & $\omega_{4,16}=-\omega_{8,12}$ & $\omega_{5,9}=\omega_{8,12}$ & $\omega_{5,10}=-\omega_{8,11}$ & $\omega_{5,11}=\omega_{8,10}$ \\
\hline$\omega_{5,12}=-\omega_{8,9}$ & $\omega_{5,13}=\omega_{8,16}$ & $\omega_{5,14}=\omega_{8,15}$ & $\omega_{5,15}=-\omega_{8,14}$ & $\omega_{5,16}=-\omega_{8,13}$ \\
\hline$\omega_{6,9}=\omega_{8,11}$ & $\omega_{6,10}=\omega_{8,12}$ & $\omega_{6,11}=-\omega_{8,9}$ & $\omega_{6,12}=-\omega_{8,10}$ & $\omega_{6,13}=-\omega_{8,15}$ \\
\hline$\omega_{6,14}=\omega_{8,16}$ & $\omega_{6,15}=\omega_{8,13}$ & $\omega_{6,16}=-\omega_{8,14}$ & $\omega_{7,9}=-\omega_{8,10}$ & $\omega_{7,10}=\omega_{8,9}$ \\
\hline$\omega_{7,11}=\omega_{8,12}$ & $\omega_{7,12}=-\omega_{8,11}$ & $\omega_{7,13}=\omega_{8,14}$ & $\omega_{7,14}=-\omega_{8,13}$ & $\omega_{7,15}=\omega_{8,16}$ \\
\hline$\omega_{7,16}=-\omega_{8,15}$ & & & & \\
\hline
\end{tabular}

Table 1. First group of equations defining $\mathfrak{s p i n}(9)$ inside $\mathfrak{s o}(16)$.

We study spinorial connections depending on 4-forms. Again, any spinor in $\Delta_{7}^{0}$ defines a unique 4-form being a solution of the corresponding overdetermined linear system and we can apply the same construction as above. Let us formulate the results.

Proposition 9.2. For any spinor $\psi \in \Delta_{7}^{0}$ there exists a unique invariant 4-form $\mathrm{T}$ such that $\{X-2 \cdot X\lrcorner \mathrm{T}\} \cdot \psi=0$ holds for any vector $X \in \mathbb{R}^{7}$.

Theorem 9.2. Any 3 -Sasakian manifold in dimension seven admits a $\mathbb{P}^{2}$-parameter family of spinorial connections defined by 4 -forms and with parallel spinors. The spinorial holonomy group of these connections is a subgroup of $\mathrm{GL}(7, \mathbb{R})$.

\section{Appendix A. The Lie algebra $\mathfrak{s p i n}(9)$ inside $\mathfrak{s o}(16)$}

The Lie algebra $\mathfrak{s o}(16)$ of all antisymmetric matrices is parameterized by 120 parameters $\omega_{i, j}, 1 \leq i<j \leq 16$. We realize the 36 -dimensional subalgebra $\mathfrak{s p i n}(9)$ by 84 explicit equations. The first group of 56 equations involves forms of type $\omega_{8, \alpha}$ and $\omega_{i, \beta}$, where $1 \leq i<8<\alpha, \beta \leq 16$, and is given in Table 1. The second group of 28 equations involves the forms $\omega_{i, j}, \omega_{\alpha, \beta}$ for $1 \leq i, j \leq 8<\alpha, \beta \leq 16$, and is given in Table 2 Consider a 3 -form $\mathrm{T} \in \mathrm{T}\left(\mathfrak{s p i n}(9), \mathbb{R}^{16}\right)$ in the antisymmetric prolongation of the $\mathfrak{s p i n}(9)$ representation in $\mathbb{R}^{16}$. Then the 2 -forms $\left.\left.\left.\left.e_{1}\right\lrcorner \mathrm{T}, e_{8}\right\lrcorner \mathrm{T}, e_{9}\right\lrcorner \mathrm{T}, e_{16}\right\lrcorner \mathrm{T}$ are elements of $\mathfrak{s p i n}(9)$. Using the first equation $\omega_{1,9}=\omega_{8,16}$ defining this subalgebra, we conclude that

$$
\mathrm{T}_{1,8,9}=0, \quad \mathrm{~T}_{1,8,16}=0, \quad \mathrm{~T}_{8,9,16}=0, \quad \mathrm{~T}_{1,9,16}=0
$$

In a similar way, the first 56 equations defining $\mathfrak{s p i n}(9)$ yield that, for $1 \leq i, j<8$ and $8<\alpha, \beta \leq 16$, the following components of $\mathrm{T}$ vanish,

$$
\mathrm{T}_{i, 8, \alpha}=0, \quad \mathrm{~T}_{8, \alpha, \beta}=0 .
$$

The second 28 equations immediately imply now that $\mathrm{T}_{i, j, 8}=0$, i.e., the interior product $\left.e_{8}\right\lrcorner \mathrm{T}=0$ vanishes for any 3 -form in the antisymmetric prolongation. Since the group $\operatorname{Spin}(9)$ acts transitively on the sphere in $\mathbb{R}^{16}$, we conclude that $\mathrm{T}=0$. 


\begin{tabular}{|l|l|}
\hline $2 \cdot \omega_{1,2}=\omega_{11,12}+\omega_{13,14}-\omega_{15,16}+\omega_{9,10}$ & $2 \cdot \omega_{1,3}=-\omega_{10,12}+\omega_{13,15}+\omega_{14,16}+\omega_{9,11}$ \\
\hline $2 \cdot \omega_{1,4}=\omega_{10,11}+\omega_{13,16}-\omega_{14,15}+\omega_{9,12}$ & $2 \cdot \omega_{1,5}=-\omega_{10,14}-\omega_{11,15}-\omega_{12,16}+\omega_{9,13}$ \\
\hline $2 \cdot \omega_{1,6}=\omega_{10,13}-\omega_{11,16}+\omega_{12,15}+\omega_{9,14}$ & $2 \cdot \omega_{1,7}=\omega_{10,16}+\omega_{11,13}-\omega_{12,14}+\omega_{9,15}$ \\
\hline $2 \cdot \omega_{1,8}=-\omega_{10,15}+\omega_{11,14}+\omega_{12,13}+\omega_{9,16}$ & $2 \cdot \omega_{2,3}=\omega_{10,11}-\omega_{13,16}+\omega_{14,15}+\omega_{9,12}$ \\
\hline $2 \cdot \omega_{2,4}=\omega_{10,12}+\omega_{13,15}+\omega_{14,16}-\omega_{9,11}$ & $2 \cdot \omega_{2,5}=\omega_{10,13}+\omega_{11,16}-\omega_{12,15}+\omega_{9,14}$ \\
\hline $2 \cdot \omega_{2,6}=\omega_{10,14}-\omega_{11,15}-\omega_{12,16}-\omega_{9,13}$ & $2 \cdot \omega_{2,7}=\omega_{10,15}+\omega_{11,14}+\omega_{12,13}-\omega_{9,16}$ \\
\hline $2 \cdot \omega_{2,8}=\omega_{10,16}-\omega_{11,13}+\omega_{12,14}+\omega_{9,15}$ & $2 \cdot \omega_{3,4}=\omega_{11,12}-\omega_{13,14}+\omega_{15,16}+\omega_{9,10}$ \\
\hline $2 \cdot \omega_{3,5}=-\omega_{10,16}+\omega_{11,13}+\omega_{12,14}+\omega_{9,15}$ & $2 \cdot \omega_{3,6}=\omega_{10,15}+\omega_{11,14}-\omega_{12,13}+\omega_{9,16}$ \\
\hline $2 \cdot \omega_{3,7}=-\omega_{10,14}+\omega_{11,15}-\omega_{12,16}-\omega_{9,13}$ & $2 \cdot \omega_{3,8}=\omega_{10,13}+\omega_{11,16}+\omega_{12,15}-\omega_{9,14}$ \\
\hline $2 \cdot \omega_{4,5}=\omega_{10,15}-\omega_{11,14}+\omega_{12,13}+\omega_{9,16}$ & $2 \cdot \omega_{4,6}=\omega_{10,16}+\omega_{11,13}+\omega_{12,14}-\omega_{9,15}$ \\
\hline $2 \cdot \omega_{4,7}=-\omega_{10,13}+\omega_{11,16}+\omega_{12,15}+\omega_{9,14}$ & $2 \cdot \omega_{4,8}=-\omega_{10,14}-\omega_{11,15}+\omega_{12,16}-\omega_{9,13}$ \\
\hline $2 \cdot \omega_{5,6}=-\omega_{11,12}+\omega_{13,14}+\omega_{15,16}+\omega_{9,10}$ & $2 \cdot \omega_{5,7}=\omega_{10,12}+\omega_{13,15}-\omega_{14,16}+\omega_{9,11}$ \\
\hline $2 \cdot \omega_{5,8}=-\omega_{10,11}+\omega_{13,16}+\omega_{14,15}+\omega_{9,12}$ & $2 \cdot \omega_{6,7}=\omega_{10,11}+\omega_{13,16}+\omega_{14,15}-\omega_{9,12}$ \\
\hline $2 \cdot \omega_{6,8}=\omega_{10,12}-\omega_{13,15}+\omega_{14,16}+\omega_{9,11}$ & $2 \cdot \omega_{7,8}=\omega_{11,12}+\omega_{13,14}+\omega_{15,16}-\omega_{9,10}$ \\
\hline
\end{tabular}

Table 2. Second group of equations defining $\mathfrak{s p i n}(9)$ inside $\mathfrak{s o}(16)$.

Proposition A.1. The antisymmetric prolongation of the unique irreducible 16-dimensional of the Lie algebra $\mathfrak{s p i n}(9)$ vanishes,

$$
\mathrm{T}\left(\mathfrak{s p i n}(9), \mathbb{R}^{16}\right)=0 .
$$

\section{References}

[1] I. Agricola, Connections on naturally reductive spaces, their Dirac operator and homogeneous models in string theory, Comm. Math. Phys. 232 (2003), 535-563.

[2] H. Baum, Th. Friedrich, R. Grunewald, I. Kath, Twistors and Killing spinors on Riemannian manifolds, Teubner-Texte zur Mathematik, Band 124, Teubner-Verlag Stuttgart / Leipzig, 1991.

[3] J. M. Bismut, A local index theorem for non-Kählerian manifolds, Math. Ann. 284 (1989), 681699.

[4] C. P. Boyer and K. Galicki, 3-Sasakian manifolds, in Essays on Einstein manifolds, (ed. by C. LeBrun and M. Wang), International Press 1999.

[5] F. M. Cabrera, M. D. Monar, A. F. Swann, Classification of $G_{2}$-structures, Journ. Lond. Math. Soc. II. Ser. 53 (1996), 407-416.

[6] E. Cartan, Sur une généralisation de la notion de courbure de Riemann et les espaces à torsion, C. R. Ac. Sc. 174 (1922), 593-595.

[7] __ Sur les variétés à connexion affine et la théorie de la relativité généralisée (première partie), Ann. Ec. Norm. Sup. 40 (1923), 325-412 et Ann. Ec. Norm. Sup. 41 (1924), 1-25.

[8] __ Sur les variétés à connexion affine et la théorie de la relativité généralisée (deuxième partie), Ann. Ec. Norm. Sup. 42 (1925), 17-88.

[9] _ Les récentes généralisations de la notion d'espace, Bull. Sc. Math.48 (1924), 294-320.

[10] M. Fernandez, A classification of Riemannian manifolds with structure group $\operatorname{Spin}(7)$, Ann. Mat. Pura Appl. 143 (1986), 101-122.

[11] M. Fernandez and A. Gray, Riemannian manifolds with structure group $\mathrm{G}_{2}$, Ann. Mat. Pura Appl. 132 (1982), 19-45. 
[12] Th. Friedrich, Der erste Eigenwert des Dirac Operators einer kompakten Riemannschen Mannigfaltigkeit nichtnegativer Skalarkrümmung, Math. Nachr. 97 (1980), 117-146.

[13] _ Dirac operators in Riemannian geometry, Graduate Studies in Mathematics 25, AMS, Privence, Rhode Island, 2000.

[14] On types of non-integrable geometries, math.dg/0205149 to appear in Suppl. Rend. Circ. Mat. di Palermo 2002.

[15] Th. Friedrich and S. Ivanov, Parallel spinors and connections with skew-symmetric torsion in string theory, Asian Journ. Math. 6 (2002), 303-336.

[16] Almost contact manifolds, connections with torsion and parallel spinors, to appear in Crelle Journal.

[17] _ Killing spinor equations in dimension 7 and geometry of integrable $\mathrm{G}_{2}$-manifolds, to appear in Journ. Geom. Phys.

[18] Th. Friedrich and I. Kath, Compact seven-dimensional manifolds with Killing spinors, Comm. Math. Phys. 133 (1990), 543-561.

[19] Th. Friedrich, I. Kath, A. Moroianu and U. Semmelmann, On nearly parallel $\mathrm{G}_{2}$-structures, Journ. Geom. Phys. 23 (1997), 256-286.

[20] S. Kobayashi and K. Nomizu, Foundations of differential geometry I, Wiley 1963.

[21] _ Foundations of differential geometry II, Wiley 1969.

[22] A. Lichnerowicz, Spin manifolds, Killing spinors and universality of the Hijazi inequality, Lett. Math. Phys. 13 (1987), 331-344.

[23] A. Trautman, On the structure of the Einstein-Cartan equations, Symp. Math. 12 (1973), 139-162.

[24] F. Tricerri and L. Vanhecke, Homogeneous structures on Riemannian manifolds, London Math. Soc. Lecture Notes Series 83, Cambridge Univ. Pres, 198x.

agricola@mathematik.hu-berlin.de

friedric@mathematik.hu-berlin.de

INSTITUT FÜR MATHEMATIK

Humboldt-UNIVERsität ZU BERLIN

UNTER DEN LINDEN 6

Sitz: John-Von-Neumann-Haus, Adlershof

D-10099 Berlin, Germany 